\title{
Practical Guidance for Interventions in Adults with Metabolic Syndrome: Diet and Exercise vs. Changes in Body Composition
}

\author{
Enrique Albert Pérez ${ }^{1}$, Marina Poveda González ${ }^{1}$, Rosa María Martínez-Espinosa ${ }^{2}$, \\ Mariola D Molina Vila ${ }^{3}$ (i) and Manuel Reig García-Galbis ${ }^{4, *(1)}$ \\ 1 Faculty of Health Sciences, University of Alicante, 03690 Alicante, Spain; ejalbertperez@gmail.com (E.A.P.); \\ marina.poveda94@gmail.com (M.P.G.) \\ 2 Division of Biochemistry and Molecular Biology, Department of Agrochemistry and Biochemistry, \\ Faculty of Sciences. University of Alicante, 03690 Alicante, Spain; rosa.martinez@ua.es \\ 3 Department of Mathematics, Faculty of Sciences, University of Alicante, 03690 Alicante, Spain; \\ mariola.molina@ua.es \\ 4 Department of Nutrition and Dietetics, Faculty of Health Sciences, University of Atacama, Avda. \\ Copayapu 2862, III Region, Copiapó 1530000, Chile \\ * Correspondence: manuel.reig@uda.cl; Tel.: +52-225-5647
}

Received: 30 May 2019; Accepted: 2 September 2019; Published: 18 September 2019

\begin{abstract}
Objective: to establish practical guidance for the design of future clinical trials in MS (metabolic syndrome) patients aged 18 and older, based on a systematic review of randomized clinical trials connecting diet, physical exercise and changes in body composition. (2) Method: this systematic review of randomized clinical trials (RCT) is based on the guidelines recommended by PRISMA (Preferred Reporting Items for Systematic reviews and Meta-Analyses). Criteria of selection: $\geq 18$ years of age; patients diagnosed with MS; intervention programs including diet, physical exercise and/or modifications in the style of life as treatment, as well as the magnitude of changes in body composition (BC); randomized clinical trial published between 2004 and 2018. (3) Results: the multidisciplinary interventions describe major changes in $\mathrm{BC}$, and the recurring pattern in these clinical trials is an energy reduction and control in the percentage of intake of macronutrients along with the performance of regularly structured exercise; the most analyzed parameter was waist circumference ( $88.9 \%$ of the trials), followed by body weight $(85.2 \%)$, BMI $(77.8 \%)$ and body fat (55.6\%). (4) Conclusions: The analysis of the information here reported sheds light for the design of future clinical trials in adults with MS. The best anthropometric parameters and units of measurement to monitor the interventions are related to dietary and physical exercise interventions. A list of practical advice that is easy to implement in daily practice in consultation is here proposed in order to guarantee the best results in changes of body composition.
\end{abstract}

Keywords: metabolic syndrome; diabetes; diet; exercise; body composition; weight and fat

\section{Introduction}

\subsection{Metabolic Syndrome (MS): Concept, Prevalence and Diagnostic Criteria}

MS was firstly described in 1920 by Kylin, a Swedish physician, as a connection between hypertension, hyperglycemia and gout. Later, in 1947, Vague indicated that visceral obesity is commonly associated with metabolic alterations, mentioning cardiovascular diseases and type 2 diabetes mellitus (T2DM) as the key pathologies. Then in 1965, at the annual meeting of the European Association for the Study of Diabetes, MS was described as a syndrome related to hypertension, hyperglycemia and obesity. 
In 1989, Kaplan linked the syndrome to the combination of upper-body obesity, glucose intolerance, hypertriglyceridemia and hypertension. However, in 1998, a WHO diabetes research group defined MS as a group of interconnected physiological, biochemical, clinical and metabolic factors that directly increase the risk of cardiovascular disease, T2DM and all-cause mortality [1]. The MS is also known as "insulin resistance syndrome", "quartet of death" or "syndrome X" [2], thus including abdominal obesity, prehypertension or hypertension, dyslipidemia and prediabetes [3]. Useful definitions for the readers to distinguish between diabetes, prediabetes, insulin resistance, etc. can be found in the review of the American Diabetes Association [4].

This syndrome affects to more than $20 \%$ of the adult population of the United States, China, Europe and the developed countries. This prevalence increases with age in a specific correlation with sex: before the age of 50 years, men have a higher prevalence and after 50 years, this trend is reversed [5-7]. There are up to five types of diagnostic criteria to identify MS [1,8], consequently the lack of unification in the universally accepted diagnostic criteria makes it difficult to determine the prevalence of MS $[9,10]$. Thus, the diagnostic criteria of the MS change depending on the age (few criteria exist for MS diagnosis of children, teenagers and adults) and the continent $[1,8,11]$.

\subsection{MS: Causes, Diet, Exercise and Anthropometric Parameters}

The main factors promoting MS and consequently T2DM are: excess ingestion of nutrients, low physical activity and the production of inflammatory cytokines [12-16]. As an example, in an investigation including 61,239 men and 73,216 women (40-74 years of age) from Shanghai (China), it was observed that with major fulfilment of the dietetic directives, minor mortality was detected [17]. The increase of physical exercise is the best non-pharmacological treatment for obesity, as it can reduce insulin resistance, to counteract the inflammatory state and to improve the lipid profile $[7,18]$. Changes of the following anthropometrical parameters could contribute to the improvement of health in MS patients: fat mass index (fat mass.height ${ }^{-2}$ ), waist circumference, abdominal diameter and corporal weight [19-22].

This systematic review is justified by the following facts:

1. The high rates of MS worldwide and the lack of consensus in the criteria used for MS diagnosis make difficult to determine MS prevalence $[1,8]$.

2. Dietary and physical exercise interventions are both causative and controlling tools for eliminating MS risk factors and the emergence of T2DM [11-15]. Thus, it is important to analyze the characteristics of the interventions causing the greatest changes [19-22].

The main objective of this systematic review was to establish practical guidance for the design of future clinical trials in MS patients aged 18 and older, based on a systematic review of randomized clinical trials connecting diet, physical exercise and changes in body composition. Other secondary objectives related to MS have also been analyzed: (a) to record which interventions (exclusive or multidisciplinary interventions) produce the greatest changes in body composition in MS patients $\geq 18$ years of age; (b) to identify which are the dietetic and physical exercise patterns showing the most significant changes in corporal composition in MS patients $\geq 18$ years of age, in order to stablish accurate clinical trials in the next future; (c) to identify the most used anthropometric parameters and units of measure to record the changes in body composition, in MS patients $\geq 18$ years old; (d) to analyze dietary and physical exercise patterns proposed by guidelines for intervention in overweight, obesity, diabetes and MS patients $\geq 18$ years of age.

\section{Materials and Methods}

\subsection{Search Strategy and Information Processing}

This systematic review of randomized clinical trials is based on the guidelines recommended by PRISMA (Preferred Reporting Items for Systematic reviews and Meta-Analyses) [23]. The information retrieval system "Boolean" was used to identify the works of interest for this review [24]. 
All the data used in this study were obtained from the following databases: CINAHL (Current Nursing and Allied Health Literature), ProQuest (which supplies services of information for universities, schools, public companies, corporations and public libraries worldwide), PubMed (a search engine for free access to the MEDLINE database of citations and abstracts of biomedical research articles) and Web of Science (online scientific information service, provided by Thomson Reuters, integrated in ISI Web of Knowledge, WOK, containing original articles based on clinical trials). The keywords used were: "MS" "weight loss", "fat loss", "diet", "exercise" and "lifestyle". These keywords were obtained from the "MeSH database" (Medical Subject Heading) and NLM (The National Library of Medicine). Controlled vocabulary thesaurus was used for indexing articles for PubMed. The search strategies used are displayed in Table 1. To identify and to select the information obtained from databases, the following filters were used: date of publication between January 2004 and December 2018. Other additional settings from each specific database were fixed: "CINAHL" database, "AB Summary" and "academic publications"; "ProQuest" database, advanced search including the options "evaluated by experts", "scientific magazines", "article" and "abstract"; "PubMed" database, advanced search including the options "Title/Abstract" and "clinical trial"; "Web of Science" database, basic search selecting the options "theme", "article" and "clinical trial". The starting open research questions were as follows. What interventions produce the greatest changes in body composition in MS patients $\geq 18$ years of age? What dietetic and physical exercise patterns show the most significant changes in corporal composition? What are the most used anthropometric parameters and units of measure to record the changes in body composition? Is it possible to propose more accurate practical guidance in order to promote more significant changes in body composition? 
Table 1. Search strategies used to identify and select clinical trials, dates: 2004-2018.

\begin{tabular}{|c|c|c|c|c|}
\hline Search Strategy & CINAHL Identified/Included & ProQuest Identified/Included & PubMed Identified/Included & Web of Science Identified/Included \\
\hline $\begin{array}{l}\text { "metabolic syndrome" AND "weight } \\
\text { loss" OR "weight reduction" OR "fat } \\
\text { loss" OR "fat reduction" OR "lifestyle" } \\
\text { AND "exercise" OR "physical activity" } \\
\text { OR "sport" OR "weightlifting" }\end{array}$ & $222 / 5$ & $532 / 7$ & $187 / 22$ & $303 / 29$ \\
\hline $\begin{array}{l}\text { "metabolic syndrome" AND "weight } \\
\text { loss" OR "weight reduction" OR "fat } \\
\text { loss" OR "fat reduction" OR "lifestyle" } \\
\text { AND "diet" OR "dietary treatment" } \\
\text { AND "feeding" OR "nutrition" OR } \\
\text { "nutritional counselling" }\end{array}$ & $27 / 1$ & $87 / 2$ & $18 / 2$ & $149 / 6$ \\
\hline $\begin{array}{l}\text { "type II diabetes" OR "insulin resistance" } \\
\text { AND "weight ls" OR "weight reduction" } \\
\text { OR "fat loss" OR "fat reduction" AND } \\
\text { "diet" OR "dietary treatment" OR } \\
\text { "feeding" AND "nutrition" OR } \\
\text { "nutritional counselling" OR "lifestyle" }\end{array}$ & $44 / 0$ & $77 / 0$ & $42 / 2$ & $155 / 3$ \\
\hline $\begin{array}{l}\text { "type II diabetes" OR "insulin resistance" } \\
\text { AND "weight loss" OR "weight } \\
\text { reduction" OR "fat loss" OR "fat } \\
\text { reduction" AND "exercise" OR "physical } \\
\text { activity" OR "sport" OR "weightlifting" }\end{array}$ & $119 / 1$ & $231 / 3$ & $152 / 4$ & $339 / 5$ \\
\hline
\end{tabular}

Articles or clinical trials identified: complete list of articles retrieved from various databases (PubMed, Web of Science, etc.), without having made the selection of the clinical trials of interest. Articles or clinical trials included: articles that meet the selection criteria. 


\subsection{Selection of the Articles Previously Identify}

Each of the identified articles were independently analyzed by three researchers. PICOS strategy was used to define the eligibility criteria for this review (population, intervention, comparisons, results and characteristics of clinical trials):

Different criteria included in the search strategy were: age of the patients $\geq 18$ years [25]; patients with MS [1,8]; intervention programs including diet, physical exercise and/or modifications in the style of life as treatment as well as the magnitude of changes in body composition; randomized clinical trial published between 2004 and 2018 in scientific journals in Spanish and English (Figure 1). The two languages were selected due to their impact and use at global scale (English is in general the "lingua franca" for communicating science. Besides, English and Spanish are two of three most spoken languages worldwide (https://danivoiceovers.com/en/los-10-idiomas-mas-habladosmundo/; https://www.europapress.es/sociedad/noticia-idiomas-cifras-cuantas-lenguas-hay-mundo20190221115202.html). The articles finally selected summarized details about the changes in body composition through the anthropometric parameters and units of measure (Tables 2 and 3) [26-62].

A total amount of 2684 articles were discarded due to the following exclusion criteria: they include one, or part of the main subjects considered for this review (MS, T2DM or insulin resistance) (1647 articles, $61.4 \%)$; diet and/or physical exercise are not included for weight loss (13 articles, $0.48 \%$ ); they are not a clinical trial, with different interventions and their comparison (589 articles, $21.94 \%$ ); they are not randomized clinical trials (six articles, $0.22 \%$ ); the decrease of body weight, BMI, body fat or waist circumference are not analyzed at least before and after the intervention (57 articles, $2.21 \%$ ); they include in the sample patients under 18 years of age (253 articles, 9.43\%); studies carried out with animal models instead of human beings (27 articles; $1.01 \%$ ); clinical trials repeated (55 articles; $2.05 \%$ ) (Figure 1) [23]. Besides, it was also necessary to discard two intervention groups from two independent clinical trials, because other methods of changes in body composition such as the gastric ball and supplementation were included as a subsection of intervention (Table 3) [48,59].

Data were extracted from the following five domains [23] (Tables 2 and 3):

1. Population: characteristics of the population studied (country of origin, type of diagnostic criteria, number, age and gender), inclusion and exclusion criteria.

2. Interventions: exclusive and multidisciplinary as therapeutic treatments.

3. Comparators: inclusion of randomized clinical trials, control and intervention groups are identified. In principle, only the intervention groups receive the therapeutic treatment that should cause changes in body composition.

4. Results: identified as variation in body composition, presenting significant and not significant variations.

5. Characteristics of clinical trials: authors, year of publication, type of randomized clinical trial, duration of intervention, instrument of analysis of body composition, type of intervention used (exclusive or multidisciplinary) and body composition variation (measured with different anthropometric parameters and units of measurement).

\subsection{Data Analysis, Identification of Information Loss Risks}

All the essential information required to carry out this systematic review is summarized in a total of six tables, a flowchart and a figure. The flowchart and Table 1 display the details of the search strategies and databases used; Tables 2 and 3 display the relevant details of the interventions recorded including the following items: title, author(s), type of sample/group, duration of the intervention, type of method/intervention, variation of the anthropometric parameters and units of measurement (this variation was registered using the following parameters): body weight (BW) in $\mathrm{kg}$; body fat (BF) in $\mathrm{kg}$ or \%; body mass index (BMI) in $\mathrm{kg} / \mathrm{m}^{2}$ and waist circumference (WC) in $\mathrm{cm}$, or their respective drop in percentage (\%) in each parameter. To identify the variation of these parameters and units, a Yes/No code has been used: YES means that the article includes the study of the parameters in the respective 
standard units, and NO means that the article does not include the parameters in their study. Thus, the most significant changes in body composition from each clinical trial was registered based on each parameter and unit (Tables 2 and 3).

The searches were made independently (one for each of the authors). The other tables were performed jointly.

Heterogeneity of the clinical trials design has been the major limitation in this research. Only few articles present adequate data to calculate heterogeneity statistics, but the contrasts done in this sense with the analyzed works reported, in general, a lack of homogeneity.

In order to analyze the quality in the design of clinical trials included in this work involving the highest variations in body composition, the Consort method has been used (assessment and implementation guide on the most appropriate guidelines for the design of randomized trials). Negative results should be considered for items that have not been performed or have been performed in an incomplete way, and positive results are those in which the items were fulfilled entirely [63]. The results of this analysis have been summarized with the calculation of the percentage of negative results compared to the totality of analyzed items. The designs in this review indicate low positive results so this would be one of the limitations of this research.

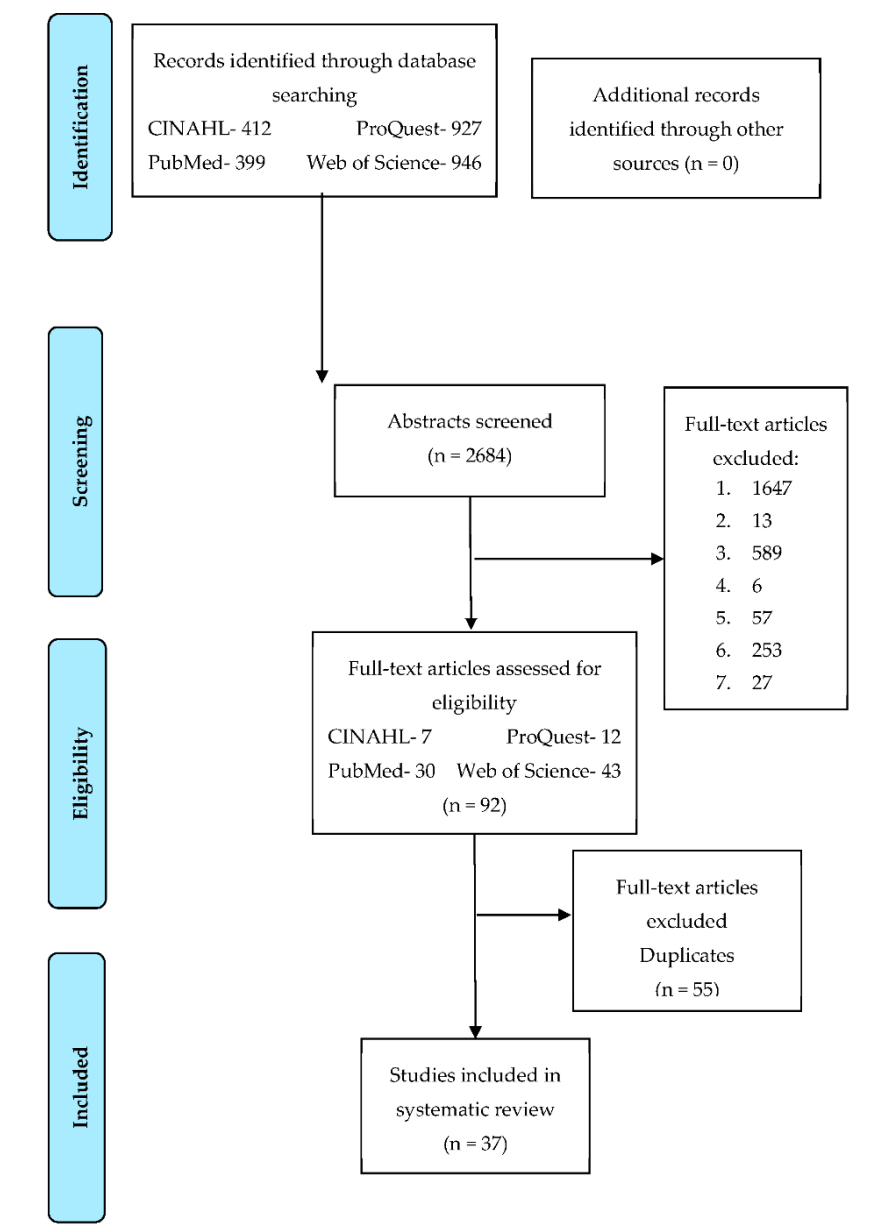

Figure 1. Flow chart in the screening process for the selection of included clinical trials [23]. Legend exclusion criteria: 1 . they include one, or part of the main subjects considered for this review (MS, T2DM or insulin resistance); 2 . diet and/or physical exercise are not included for weight loss; 3 . They are not clinical trials, with different interventions and their comparison; 4 . not being a randomized clinical trial; 5 . the decrease of body weight, BMI, body fat or waist circumference are not analyzed at least before and after the intervention; 6 . they include in the sample patients under 18 years of age; 7. studies carried out with animal models instead of human beings. 
Table 2. Characteristics of the randomized trials included in the reduction of body composition in metabolic syndrome: exclusive interventions.

\begin{tabular}{|c|c|c|c|c|c|c|c|c|c|c|c|c|}
\hline \multirow{2}{*}{$\begin{array}{l}\text { Author (s) } \\
{[26-35]}\end{array}$} & \multirow{2}{*}{ Location } & \multirow{2}{*}{$\begin{array}{l}\text { Study } \\
\text { Design }\end{array}$} & \multirow{2}{*}{$\begin{array}{l}\text { MS Diagnosis } \\
\text { Criteria [1] }\end{array}$} & \multirow{2}{*}{$\begin{array}{l}\text { Sample/Groups/ } \\
\text { Characteristics Studied }\end{array}$} & \multirow{2}{*}{$\begin{array}{l}\text { Duration } \\
\text { (Months) }\end{array}$} & \multirow{2}{*}{$\begin{array}{l}\text { Body Composition } \\
\text { Measurement Instrument }\end{array}$} & \multirow{2}{*}{$\begin{array}{c}\text { Intervention Method } \\
\text { Characteristics } \\
\text { Studied/Comparative Statistical } \\
\text { Analysis of BC } \\
\end{array}$} & \multicolumn{4}{|c|}{$\begin{array}{l}\text { Anthropometric Parameters and Measurement } \\
\text { Unit Analyzed (Statistical Results) }\end{array}$} & \multirow{2}{*}{$\begin{array}{c}\text { Decreases in Body Composition } \\
\text { Mean } \pm \text { SD or Mean } \pm \text { (SE) or } \\
\text { Mean }(\mathrm{Cl}, 95 \%)\end{array}$} \\
\hline & & & & & & & & BW (kg/\%) & $\begin{array}{c}\text { BF } \\
(\mathrm{kg} \text { or \%) }\end{array}$ & $\begin{array}{c}\text { BMI } \\
\left(\mathrm{kg} / \mathrm{m}^{2} / \%\right)\end{array}$ & $\begin{array}{c}\mathrm{WC} \\
(\mathrm{cm} / \%)\end{array}$ & \\
\hline [26] & $\begin{array}{l}\text { Asia } \\
\text { (Iran) }\end{array}$ & RCT & ATPIII & $\begin{array}{l}\mathrm{n}=87 \\
\text { IG1: } 43 \\
\text { IG2: } 44 \\
\text { Women: } 60.95 \% \\
\text { Age: } 45.5\end{array}$ & 2.5 & $\begin{array}{l}\text { Body composition indices } \\
\text { were measured via } \\
\text { bioelectric impedance } \\
\text { analysis (BIA; In Body s10; } \\
\text { Korea). Height was } \\
\text { measured bar using } \\
\text { a stadiometer }\end{array}$ & $\begin{array}{l}\text { Diet } \\
\text { - Within groups: } \\
\text { IG1: low fat plain } \\
\text { yogurt } \\
\text { IG2: fortified yogurt } \\
\text { - Between groups }\end{array}$ & $\begin{array}{c}p<0.001 \\
p<0.001 \\
\quad \text { NS }\end{array}$ & $\begin{array}{l}p<0.0001 \\
p<0.05 \\
p<0.05\end{array}$ & $\begin{array}{c}p<0.001 \\
p<0.001 \\
\quad \text { NS }\end{array}$ & $\begin{array}{l}p<0.001 \\
p<0.001 \\
p<0.05\end{array}$ & $\begin{array}{c}\text { BW: } \\
\text { IG1: } 4.3 \pm 1.9 \mathrm{~kg} \\
\text { IG2: } 5.1 \pm 3.0 \mathrm{~kg} \\
\text { BF: } \\
\text { IG1: } 1.7 \pm 2.4 \mathrm{~kg} \text { and } 1.7 \pm 3.2 \% \\
\text { IG2: } 3.4 \pm 4.7 \mathrm{~kg} \text { and } 3.0 \pm 2.8 \% \\
\text { BMI } \\
\text { IG1: } 1.6 \pm 0.1 \mathrm{~kg} \\
\text { IG2: } 1.8 \pm 1.16 \mathrm{~kg} \\
\text { WC: } \\
\text { IG1: } 4.4 \pm 1.7 \mathrm{~cm} \\
\text { IG2: } 5.8 \pm 2.0 \mathrm{~cm} \\
\end{array}$ \\
\hline$[27]$ & $\begin{array}{l}\text { Asia } \\
\text { (Israel) }\end{array}$ & $\begin{array}{c}\mathrm{RCT} \\
\text { (parallel-arm) }\end{array}$ & ATPIII & $\begin{array}{c}\mathrm{n}=74 \\
\text { IG1: } 38 \\
\text { IG2: } 36 \\
\text { Women: } 100 \% \\
\text { Age: } 30-57\end{array}$ & 3 & $\begin{array}{l}\text { BW and WC were measured } \\
\text { by using a scale model } \\
\text { Detector Physician Beam } \\
\text { Scale (HOSPEQ, Inc., Miami, } \\
\text { FL) vs. the same person } \\
\text { acoording to the guidelines } \\
\text { of the National Heart, Lung, } \\
\text { and Blood } \\
\text { Institute respectively }\end{array}$ & $\begin{array}{l}\text { Diet- Within groups: } \\
\text { IG1 (high breakfast kcal content) } \\
\text { IG2 (high dinner kcal content) } \\
\text { - Between groups }\end{array}$ & $\begin{array}{c}\text { YES/NO } \\
p<0.0001 \\
p<0.05 \\
p<0.0001\end{array}$ & $\mathrm{NO} / \mathrm{NO}$ & $\begin{array}{l}\text { YES/NO } \\
p<0.0001 \\
p<0.05 \\
p<0.0001\end{array}$ & $\begin{array}{l}p<0.0001 \\
p<0.05 \\
p<0.0001\end{array}$ & $\begin{array}{c}\text { BW: } \\
\text { IG1: } 8.7 \pm(1.4) \mathrm{kg} \\
\text { IG2: } 3.6 \pm(1.5) \mathrm{kg} \\
\text { BMI: } \\
\text { IG1: } 3.1 \pm(0.4) \mathrm{kg} / \mathrm{m}^{2} \\
\text { IG2: } 1.3 \pm(0.4) \mathrm{kg} / \mathrm{m} 2 \\
\text { WC: } \\
\text { IG1: } 8.7 \pm(0.9) \mathrm{cm} \\
\text { IG2: } 3.6 \pm(0.9) \mathrm{cm} \\
\end{array}$ \\
\hline [28] & $\begin{array}{l}\text { Europe } \\
\text { (Spain) }\end{array}$ & $\begin{array}{c}\mathrm{RCT} \\
\text { (parallel-group) }\end{array}$ & ATP III & $\begin{array}{c}\mathrm{n}=50 \\
\text { IG1: } 25 \\
\text { IG2: } 25 \\
\text { Men (56\%) } \\
\text { Age: } 18-65\end{array}$ & 3 & $\begin{array}{l}\text { Height and BC was } \\
\text { measured using a } \\
\text { wall-mounted stadiometer } \\
\text { vs. Tanita TBF- } 300 \text { (Tanita } \\
\text { Corp., Tokyo, Japan) } \\
\text { bioimpedance analysis } \\
\text { device respectively }\end{array}$ & $\begin{array}{l}\text { Diet } \\
\text {-Within groups: } \\
\text { IG1 (nut diet) } \\
\text { IG2 } \\
\text { - Between groups }\end{array}$ & $\begin{array}{l}p<0.05 \\
p<0.05 \\
\quad \text { NS }\end{array}$ & $\begin{array}{l}p<0.05 \\
p<0.05 \\
\quad \text { NS }\end{array}$ & $\mathrm{NO} / \mathrm{NO}$ & $\begin{array}{l}p<0.05 \\
p<0.05 \\
\quad \text { NS }\end{array}$ & $\begin{array}{c}\text { BW: } \\
\text { IG1: } 2.2 \mathrm{~kg} \\
(0.9-3.4) \\
\text { IG2: } 1.5 \mathrm{~kg} \\
(0.6-2.4) \\
\text { BF: } \\
\text { IG1: } 1.9 \%(0.9-2.5) \\
\text { IG2: } 1.1 \%(0.3-1.9) \\
\text { WC: } \\
\text { IG1: } 3.8 \mathrm{~cm} \\
(1.9-5.8) \\
\text { IG2: } 2.7 \mathrm{~cm} \\
(1.0-4.4)\end{array}$ \\
\hline
\end{tabular}


Table 2. Cont.

\begin{tabular}{|c|c|c|c|c|c|c|c|c|c|c|c|c|}
\hline \multirow{2}{*}{$\begin{array}{l}\text { Author (s) } \\
{[26-35]}\end{array}$} & \multirow{2}{*}{ Location } & \multirow{2}{*}{$\begin{array}{l}\text { Study } \\
\text { Design }\end{array}$} & \multirow{2}{*}{$\begin{array}{l}\text { MS Diagnosis } \\
\text { Criteria [1] }\end{array}$} & \multirow{2}{*}{$\begin{array}{l}\text { Sample/Groups/ } \\
\text { Characteristics Studied }\end{array}$} & \multirow{2}{*}{$\begin{array}{l}\text { Duration } \\
\text { (Months) }\end{array}$} & \multirow{2}{*}{$\begin{array}{c}\text { Body Composition } \\
\text { Measurement Instrument }\end{array}$} & \multirow{2}{*}{$\begin{array}{c}\text { Intervention Method } \\
\text { Characteristics } \\
\text { Studied/Comparative Statistical } \\
\text { Analysis of BC }\end{array}$} & \multicolumn{4}{|c|}{$\begin{array}{l}\text { Anthropometric Parameters and Measurement } \\
\text { Unit Analyzed (Statistical Results) }\end{array}$} & \multirow{2}{*}{$\begin{array}{c}\text { Decreases in Body Composition } \\
\text { Mean } \pm \text { SD or Mean } \pm \text { (SE) or } \\
\text { Mean }(\mathrm{CI}, 95 \%)\end{array}$} \\
\hline & & & & & & & & BW (kg/\%) & $\begin{array}{c}\mathrm{BF} \\
(\mathrm{kg} \text { or } \%)\end{array}$ & $\begin{array}{c}\text { BMI } \\
\left(\mathrm{kg} / \mathrm{m}^{2} / \%\right)\end{array}$ & $\begin{array}{c}\mathrm{WC} \\
(\mathrm{cm} / \%)\end{array}$ & \\
\hline [29] & $\begin{array}{l}\text { Europe } \\
\text { (Italy) }\end{array}$ & RCT & ATP III & $\begin{array}{c}\mathrm{n}=100 \\
\text { IG1: } 50 \\
\text { IG2: } 50 \\
\text { Women: } 73 \% \\
\text { Age: }>18\end{array}$ & 5 & $\begin{array}{l}\text { Height was measured using } \\
\text { a stadiometer and WC, to the } \\
\text { measurement of the } \\
\text { narrowest circumference } \\
\text { between the bottom of the } \\
\text { rib cage and the iliac crest by } \\
\text { using an unstretched } \\
\text { tape measure }\end{array}$ & $\begin{array}{l}\text { Diet } \\
\text { - Within groups: } \\
\text { IG1 (high CH diet) } \\
\text { IG2 (low CH diet) } \\
\text { - Between groups }\end{array}$ & $\begin{array}{l}p<0.001 \\
p<0.001 \\
\quad \text { NS }\end{array}$ & $\mathrm{NO} / \mathrm{NO}$ & $\begin{array}{l}p<0.001 \\
p<0.001 \\
\quad \text { NS }\end{array}$ & $\begin{array}{l}p<0.001 \\
p<0.001 \\
\quad \text { NS }\end{array}$ & $\begin{array}{c}\text { BW: } \\
\text { IG1: NE (kg) } \\
\text { IG1: } 10 \% \\
\text { I22: NE (kg) } \\
\text { IG2: } 10 \% \\
\text { BMI: } \\
\text { IG1: NE } \\
\text { IG2: NE } \\
\text { WC: } \\
\text { IG1: NE (cm) } \\
\text { IG1: } 8 \% \\
\text { IG2: } \% \text { (cm) } \\
\text { IG2: } \%\end{array}$ \\
\hline [30] & $\begin{array}{l}\text { Europe } \\
\text { (Spain) }\end{array}$ & $\begin{array}{c}\mathrm{RCT} \\
\text { (Randomized-block) }\end{array}$ & IDF & $\begin{array}{c}\mathrm{n}=160 \\
\text { IG: } 138 \\
\text { CG: } 22 \\
\text { Women: } 50 \% \\
\text { Age: } 54 \pm 8\end{array}$ & 4 & $\begin{array}{l}\text { Body weight was assessed in } \\
\text { an electronic scale (Hawk, } \\
\text { Mettler Toledo, USA) body } \\
\text { composition was determined } \\
\text { by dual energy X-ray } \\
\text { absorptiometry (DXA } \\
\text { Hologic Series Discovery Wi } \\
\text { QDR, Bedford, USA). WC } \\
\text { was measured in a } \\
\text { horizontal plane } 2 \text { cm above } \\
\text { the iliac crest }\end{array}$ & $\begin{array}{l}\text { Exercise } \\
\text { Within groups: } \\
\text { IG: aerobic interval training } \\
\text { CG: sedentary } \\
\text { Between groups }\end{array}$ & $\begin{array}{l}p<0.05 \\
\quad \mathrm{NE} \\
p<0.05\end{array}$ & $\begin{array}{l}\text { NS } \\
\text { NE } \\
\text { NS }\end{array}$ & $\begin{array}{l}p<0.05 \\
\quad \mathrm{NE} \\
p<0.05\end{array}$ & $\begin{array}{l}p<0.05 \\
\quad \mathrm{NE} \\
p<0.05\end{array}$ & $\begin{array}{c}\text { BW: } \\
\text { IG: } 1.2 \mathrm{~kg} \\
\text { CG: increase } \\
\text { BF: } \\
\text { IG: } 0.7 \mathrm{~kg} \\
\text { CG: increase } \\
\text { BMI: } \\
\text { IG: } 0.4 \mathrm{~kg} \\
\text { CG: increase } \\
\text { WC: } \\
\text { IG: } 2.6 \mathrm{~cm} \\
\text { CG: increase }\end{array}$ \\
\hline [31] & $\begin{array}{l}\text { North } \\
\text { America } \\
\text { (USA) }\end{array}$ & RCT & IDF & $\begin{array}{c}\mathrm{n}=34 \\
\text { CG: } 10 \\
\text { IG1:13 } \\
\text { IG2: } 11 \\
\text { Women: } 75 \% \\
\text { Age: } 49.1 \pm 1.8\end{array}$ & 4 & $\begin{array}{l}\text { Air displacement } \\
\text { plethysmography (Bod-Pod; } \\
\text { Life Measurement } \\
\text { Instruments, Concord, CA) }\end{array}$ & $\begin{array}{l}\text { Exercise } \\
\text { - Within groups: } \\
\text { IG1 (low intensity exercise) } \\
\text { IG2 (high intensity) } \\
\text { - Between groups }\end{array}$ & $\begin{array}{l}p<0.01 \\
p<0.01 \\
\text { NS }\end{array}$ & $\begin{array}{l}p<0.01 \\
p<0.01 \\
\text { NS }\end{array}$ & $\begin{array}{l}p<0.01 \\
p<0.01 \\
\text { NS }\end{array}$ & $\begin{array}{l}\text { NS } \\
p<0.01 \\
\text { NS }\end{array}$ & $\begin{array}{c}\text { BW: } \\
\text { IG1: } 2.7 \mathrm{~kg} \\
\text { IG2: } 2.7 \mathrm{~kg} \\
\text { BF: } \\
\text { IG1: } 0.7 \% \\
\text { IG2: } 1.6 \% \\
\text { BMI: } \\
\text { IG1: } 0.9 \mathrm{~kg} / \mathrm{m}^{2} \\
\text { IG2: } 0.9 \mathrm{~kg} / \mathrm{m}^{2} \\
\text { WC: } \\
\text { IG1: } 2 \mathrm{~cm} \\
\text { IG2: } 4.9 \mathrm{~cm}\end{array}$ \\
\hline [32] & $\begin{array}{l}\text { North } \\
\text { America } \\
\text { (USA) }\end{array}$ & RCT & IDF & $\begin{array}{l}\mathrm{n}=27 \\
\text { CG: } \\
\text { IG1: } 11 \\
\text { IG2: } 9 \\
\text { Women: } 100 \% \\
\text { Age: } 51 \pm 9\end{array}$ & 4 & $\begin{array}{l}\text { Air displacement } \\
\text { plethysmography (Bod-Pod, } \\
\text { Life Measurement } \\
\text { Instruments, Concord, CA) }\end{array}$ & $\begin{array}{l}\text { Exercise } \\
\text { - Within groups: } \\
\text { IG1 (low-intensity aerobic) } \\
\text { IG2 (moderate-to-high intensity } \\
\text { aerobic) } \\
\text { - Between groups: } \\
\text { IG1 vs. IG2 } \\
\text { IG1 vs. CG } \\
\text { IG2 vs. CG }\end{array}$ & $\begin{array}{c}\text { NS } \\
p<0.05 \\
\text { NS } \\
\text { NS } \\
\text { NS }\end{array}$ & $\begin{array}{c}\begin{array}{c}\text { YES } \\
(\%) / \mathrm{NO}\end{array} \\
\text { NS } \\
p<0.05 \\
\text { NS } \\
\text { NS } \\
\text { NS }\end{array}$ & $\begin{array}{c}\text { NS } \\
p<0.05 \\
\text { NS } \\
\text { NS } \\
\text { NS }\end{array}$ & $\begin{array}{c}\text { NS } \\
p<0.05 \\
p<0.05 \\
\text { NS } \\
p<0.05\end{array}$ & $\begin{array}{c}\text { BW: } \\
\text { IG1: } 2.1 \mathrm{~kg} \\
\text { IG2: } 3.5 \mathrm{~kg} \\
\text { BF: } \\
\text { IG1: } 0.4 \% \\
\text { IG2: } 1.7 \% \\
\text { BMI: } \\
\text { IG1: } 0.8 \mathrm{~kg} / \mathrm{m}^{2} \\
\text { IG2: } 1.3 \mathrm{~kg} / \mathrm{m}^{2} \\
\text { WC: } \\
\text { IG1: } 1.2 \mathrm{~cm} \\
\text { IG2: } 5.6 \mathrm{~cm}\end{array}$ \\
\hline
\end{tabular}


Table 2. Cont.

\begin{tabular}{|c|c|c|c|c|c|c|c|c|c|c|c|c|}
\hline \multirow{2}{*}{$\begin{array}{l}\text { Author (s) } \\
{[26-35]}\end{array}$} & \multirow{2}{*}{ Location } & \multirow{2}{*}{$\begin{array}{l}\text { Study } \\
\text { Design }\end{array}$} & \multirow{2}{*}{$\begin{array}{l}\text { MS Diagnosis } \\
\text { Criteria [1] }\end{array}$} & \multirow{2}{*}{$\begin{array}{l}\text { Sample/Groups/ } \\
\text { Characteristics Studied }\end{array}$} & \multirow{2}{*}{$\begin{array}{l}\text { Duration } \\
\text { (Months) }\end{array}$} & \multirow{2}{*}{$\begin{array}{c}\text { Body Composition } \\
\text { Measurement Instrument }\end{array}$} & \multirow{2}{*}{$\begin{array}{c}\text { Intervention Method } \\
\text { Characteristics } \\
\text { Studied/Comparative Statistical } \\
\text { Analysis of BC }\end{array}$} & \multicolumn{4}{|c|}{$\begin{array}{l}\text { Anthropometric Parameters and Measurement } \\
\text { Unit Analyzed (Statistical Results) }\end{array}$} & \multirow{2}{*}{$\begin{array}{c}\text { Decreases in Body Compositio } \\
\text { Mean } \pm \text { SD or Mean } \pm \text { (SE) or } \\
\text { Mean (CI, } 95 \%)\end{array}$} \\
\hline & & & & & & & & BW (kg/\%) & $\begin{array}{c}\mathrm{BF} \\
(\mathrm{kg} \text { or } \%)\end{array}$ & $\begin{array}{c}\text { BMI } \\
\left(\mathrm{kg} / \mathrm{m}^{2} / \%\right)\end{array}$ & $\begin{array}{c}\mathrm{WC} \\
(\mathrm{cm} / \%)\end{array}$ & \\
\hline [33] & $\begin{array}{c}\text { Asia } \\
\text { (China) }\end{array}$ & RCT & IDF & $\begin{array}{c}\mathrm{n}=173 \\
\text { IG: } 86 \\
\text { CG: } 87 \\
\text { Women: } 50.8 \% \\
\text { Age: } 24-78\end{array}$ & 3 & $\mathrm{NE}$ & $\begin{array}{l}\text { Intervention in lifestyle } \\
\text { - Within groups: } \\
\text { IG (Lifestyle intervention } \\
\text { program) } \\
\text { - Between groups }\end{array}$ & $\begin{array}{c}\mathrm{NE} \\
p<0.01\end{array}$ & $\mathrm{NO} / \mathrm{NO}$ & $\begin{array}{c}\text { NE } \\
p<0.01\end{array}$ & YES/NO & $\begin{array}{c}\text { BW: } \\
\text { IG: } 1.77 \mathrm{~kg} \\
\text { CG: increase } \\
\text { BMI: } \\
\text { IG: } 0.58 \mathrm{~kg} / \mathrm{m}^{2} \\
\text { CG: } 0.01 \mathrm{~kg} / \mathrm{m}^{2} \\
\text { WC: } \\
\text { IG: } 3.69 \text { (male) } / 1.37 \text { (female) } \\
\text { CG: } 1.61 \text { (male) } / 1.34 \text { (female) }\end{array}$ \\
\hline [34] & $\begin{array}{l}\text { North } \\
\text { America } \\
\text { (USA) }\end{array}$ & RCT & IDF & $\begin{array}{l}\mathrm{n}=135 \\
\text { IG1: } 72 \\
\text { IG2: } 63 \\
\text { Women: } 75 \% \\
\text { Age: } 52\end{array}$ & 24 & $\begin{array}{l}\text { The International Diabetes } \\
\text { Federation definition } \\
\text { requires central obesity, } \\
\text { measured by WC with } \\
\text { ethnicity-based cutoffs }\end{array}$ & $\begin{array}{l}\text { Intervention in lifestyle } \\
\text { - Within groups: } \\
\text { IG1 (individual counselling) } \\
\text { IG2 (group counselling) } \\
\text { - Between groups }\end{array}$ & $\begin{array}{l}p<0.05 \\
p<0.05 \\
p<0.001\end{array}$ & $\mathrm{NO} / \mathrm{NO}$ & $\begin{array}{l}p<0.001 \\
p<0.05 \\
p<0.05\end{array}$ & $\begin{array}{c}p<0.05 \\
p<0.05 \\
\text { NS }\end{array}$ & $\begin{array}{c}\text { BW: } \\
\text { IG1: } 2.2 \pm 14.2 \mathrm{~kg} \\
\text { IG1: } 1.8 \pm 18.6 \% \\
\text { IG2: } 6.2 \pm 14.3 \mathrm{~kg} \\
\text { IG2: } 5.6 \pm 26.8 \% \\
\text { BMI: } \\
\text { IG1: } 0.8 \mathrm{~kg} / \mathrm{m}^{2} \\
\text { IG2: } 2.1 \mathrm{~kg} / \mathrm{m}^{2} \\
\text { WC: } \\
\text { IG1: } 2.4 \pm 15.5 \mathrm{~cm} \\
\text { IG2: } 3.1 \pm 15.5 \mathrm{~cm}\end{array}$ \\
\hline [35] & $\begin{array}{c}\text { Asia } \\
\text { (East Asia) }\end{array}$ & RCT & $\begin{array}{l}\text { The Examination } \\
\text { Committee of } \\
\text { Criteria } \\
\text { for "Metabolic } \\
\text { Syndrome" in Japan }\end{array}$ & $\begin{array}{c}\mathrm{n}=102 \\
\text { IG:49 } \\
\text { CG:53 } \\
\text { Men: } 100 \% \\
\text { Age: } 53.2 \pm 6.8\end{array}$ & 6 & $\begin{array}{l}\text { Body height and body } \\
\text { weight were measured using } \\
\text { an automated scale } \\
\text { (AD-6225A; A\&D, } \\
\text { Tokyo, Japan) }\end{array}$ & $\begin{array}{l}\text { Intervention in lifestyle } \\
\text { CG (standard healthy } \\
\text { recommendations) } \\
\text { - Within groups: } \\
\text { IG (intervention in lifestyle) } \\
\text { - Between groups }\end{array}$ & $\begin{array}{l}p<0.001 \\
p<0.001\end{array}$ & $\mathrm{NO} / \mathrm{NO}$ & $\begin{array}{l}p<0.001 \\
p<0.01\end{array}$ & $\begin{array}{l}p<0.001 \\
p<0.05\end{array}$ & $\begin{array}{c}\text { BW: } \\
\text { IG: } 2.01 \mathrm{~kg} \\
\text { BMI: } \\
\text { IG: } 0.6 \mathrm{~kg} / \mathrm{m}^{2} \\
\text { WC: } \\
\text { IG: } 2.51 \mathrm{~cm}\end{array}$ \\
\hline
\end{tabular}

Body weight (BW); body fat (BF); body mass index (BMI); waist circumference (WC); control group (CG); intervention group (IG); carbohydrates (CH); non-significant (NS); the information is not available in the clinical trial evaluated (NE); randomized controlled trial (RCT); YES: the article includes the analysis of the parameter expressed in its correspondent units; NO: the article does not include the analysis of the parameter. 
Table 3. Characteristics of the randomized trials included in the reduction of body composition in metabolic syndrome: multidisciplinary interventions.

\begin{tabular}{|c|c|c|c|c|c|c|c|c|c|c|c|c|}
\hline \multirow{2}{*}{$\begin{array}{l}\text { Author(s) } \\
{[36-62]}\end{array}$} & \multirow[t]{2}{*}{ Location } & \multirow{2}{*}{$\begin{array}{l}\text { Study } \\
\text { Design }\end{array}$} & \multirow{2}{*}{$\begin{array}{l}\text { MS Diagnosis } \\
\text { Criteria [1] }\end{array}$} & \multirow{2}{*}{$\begin{array}{l}\text { Sample/Groups/ } \\
\text { Characteristics Studied }\end{array}$} & \multirow{2}{*}{$\begin{array}{l}\text { Duration } \\
\text { (months) }\end{array}$} & \multirow{2}{*}{$\begin{array}{c}\text { Body Composition } \\
\text { Measurement Instrument }\end{array}$} & \multirow{2}{*}{$\begin{array}{c}\text { Intervention } \\
\text { Method/Comparative Statistical } \\
\text { Analysis of the BC }\end{array}$} & \multicolumn{4}{|c|}{$\begin{array}{l}\text { Anthropometric Parameters and Measurement Unit } \\
\text { Analyzed (Statistical results) }\end{array}$} & \multirow{2}{*}{$\begin{array}{c}\text { Decreases in Body Composition } \\
\text { Mean } \pm \text { SD or Mean } \pm \text { (SE) or } \\
\text { Mean (CI, }, 55 \%)\end{array}$} \\
\hline & & & & & & & & BW (kg/\%) & BF (kg or \%) & $\begin{array}{c}\text { BMI } \\
\left(\mathrm{kg} / \mathrm{m}^{2} / \%\right)\end{array}$ & $\begin{array}{c}\text { WC } \\
(\mathrm{cm} / \%)\end{array}$ & \\
\hline [36] & $\begin{array}{l}\text { North } \\
\text { America } \\
\text { (USA) }\end{array}$ & RCT & NE & $\begin{array}{c}\mathrm{n}=39 \\
\text { IG1:23 } \\
\text { IG2:16 } \\
\text { Women: } 60 \% \\
\text { Age: } 38-76\end{array}$ & 6 & $\mathrm{NE}$ & $\begin{array}{l}\text { Diet, exercise } \\
\text { - Within groups: } \\
\text { IG1 (hypocaloric diet + MUFA) } \\
\text { IG2 hypocaloric diet + PUFA) } \\
\text { - Between groups }\end{array}$ & $\begin{array}{c}\text { YES/NO } \\
\text { NS } \\
p<0.01 \\
\text { NE }\end{array}$ & $\mathrm{NO} / \mathrm{NO}$ & $\mathrm{NO} / \mathrm{NO}$ & $\mathrm{NO} / \mathrm{NO}$ & $\begin{array}{c}\text { BW: } \\
\text { IG1: } 2.3 \mathrm{~kg} \pm(1) \\
\text { IG2: } 4.6 \mathrm{~kg} \pm(2)\end{array}$ \\
\hline [37] & $\begin{array}{l}\text { Europe } \\
\text { (Spain) }\end{array}$ & RCT & NE & $\begin{array}{c}\mathrm{n}=36 \\
\text { CG: } 12 \\
\text { IG1:12 } \\
\text { IG2: } 12 \\
\text { Men: } 75 \% \\
\text { Age: } 54 \pm 9\end{array}$ & 4 & $\begin{array}{l}\text { Dual Energy X-ray } \\
\text { absorptiometry scans } \\
\text { (Hologic Discovery DXA } \\
\text { Series Wi QDR, } \\
\text { Bedford, USA) }\end{array}$ & $\begin{array}{l}\text { Diet, exercise } \\
\text { - Within groups: } \\
\text { IG1 (EXER-then-DIET) } \\
\text { IG2 (EXER+DIET) } \\
\text { - Between groups: } \\
\text { IG1 vs. CG } \\
\text { IG2 vs. CG } \\
\text { IG2 vs. IG1 }\end{array}$ & $\begin{array}{c}\mathrm{NS} \\
p<0.05 \\
\mathrm{NE} \\
\mathrm{NE} \\
\mathrm{NE}\end{array}$ & $\begin{array}{c}\mathrm{NS} \\
p<0.05 \\
\mathrm{NE} \\
\mathrm{NE} \\
\mathrm{NE}\end{array}$ & $\begin{array}{c}\text { NS } \\
p<0.05 \\
\mathrm{NE} \\
\mathrm{NE} \\
\mathrm{NE}\end{array}$ & $\begin{array}{c}p<0.05 \\
p<0.05 \\
\text { NS } \\
p<0.05 \\
\text { NS }\end{array}$ & $\begin{array}{c}\text { BW: } \\
\text { IG1: } \mathrm{NE} \mathrm{kg} \\
\text { IG1: NE\% } \\
\text { IG2: } \mathrm{NE} \mathrm{kg} \\
\text { IG2: } 5.5 \pm 0.8 \% \\
\text { BF: } \\
\text { IG1: } \mathrm{NE}(\mathrm{kg}) \\
\text { IG2: } \mathrm{NE}(\mathrm{kg}) \\
\text { BMI: } \\
\text { IG1: increase } \\
\text { IG2: } 1.8 \mathrm{~kg} / \mathrm{m}^{2} \\
\text { WC: } \\
\text { IG1: } \mathrm{NE}(\mathrm{cm}) \\
\text { IG2: } \mathrm{NE}(\mathrm{cm}) \\
\end{array}$ \\
\hline [38] & $\begin{array}{l}\text { Oceania } \\
\text { (Australia) }\end{array}$ & RCT & ATPIII & $\begin{array}{l}n=62 \\
\text { IG1: } 21 \\
\text { IG2: } 20 \\
\text { IG3: } 21 \\
\text { Women: } 55 \% \\
\text { Age: } 30-60\end{array}$ & 6 & $\begin{array}{l}\text { The participants weighing < } \\
157 \text { kg was measured with } \\
\text { Hologic DXA (QDR-4500W; } \\
\text { Hologic Corporation), and } \\
\text { those weighing > } 177 \mathrm{~kg} \\
\text { were measured with a GE } \\
\text { Lunar iDXA } \\
\text { (General Electric) }\end{array}$ & $\begin{array}{l}\text { Diet, exercise } \\
\text { - Within groups: } \\
\text { IG1 (M-DASH diet) } \\
\text { IG2 ( (BOLD diet) } \\
\text { IG3 (BOLD + diet) } \\
\text { - Between groups }\end{array}$ & $\begin{array}{l}p<0.001 \\
p<0.001 \\
p<0.001 \\
\quad \text { NS }\end{array}$ & $\begin{array}{l}p<0.001 \\
p<0.001 \\
p<0.001 \\
\quad \text { NS }\end{array}$ & $\begin{array}{l}p<0.001 \\
p<0.001 \\
p<0.001 \\
\quad \text { NS }\end{array}$ & $\begin{array}{l}p<0.001 \\
p<0.001 \\
p<0.001 \\
\quad \text { NS }\end{array}$ & $\begin{array}{c}\text { BW: } \\
\text { IG1: } 5.1 \mathrm{~kg} \\
\text { IG2: } 4.8 \mathrm{~kg} \\
\text { IG3: } 4.8 \mathrm{~kg} \\
\text { BF: } \mathrm{NE} \\
\text { BMI: } \\
\text { IG1: } 1.8 \mathrm{~kg} / \mathrm{m}^{2} \\
\text { IG2: } 0.5 \mathrm{~kg} / \mathrm{m}^{2} \\
\text { I2: } 1.7 \mathrm{~kg} / \mathrm{m}^{2} \\
\text { WC: } \\
\text { IG1: } 5.5 \mathrm{~cm} \\
\text { IG2: } 7.6 \mathrm{~cm} \\
\text { IG3: } 6.2 \mathrm{~cm} \\
\end{array}$ \\
\hline [39] & $\begin{array}{l}\text { Europe } \\
\text { (Spain) }\end{array}$ & RCT & ATP III & $\begin{array}{l}\mathrm{n}=36 \\
\text { III1: } 8 \\
\text { IG2: } 8 \\
\text { IG3: } 10 \\
\text { IG: } 10 \\
\text { Women and Men: NE } \\
\text { Age: } 50-70\end{array}$ & 3 & $\begin{array}{l}\text { The anthropometric study } \\
\text { stated by the International } \\
\text { Society for the Advancement } \\
\text { of Kit anthropometry } \\
\text { (method ISAK); to evaluate } \\
\text { BW and height rods were } \\
\text { used (MB 201T Bonus); the } \\
\text { cutaneous folds were valued } \\
\text { by means of the } \\
\text { Harpenden calibrator }\end{array}$ & $\begin{array}{l}\text { Diet, exercise } \\
\text { Within groups: } \\
\text { IG1 (Hypocaloric Med diet) } \\
\text { IG2 (Low fat-high CH diet) } \\
\text { IG3 (Hypocaloric Med diet and } \\
\text { exercise) } \\
\text { IG4 (Low fat-high diet and } \\
\text { exercise) } \\
\text { - Between groups: } \\
\text { IG2 vs. IG1 } \\
\text { IG2 vs. IG3 } \\
\text { IG2 vs. IG4 } \\
\text { IG4 vs. IG1 } \\
\text { IG4 vs. IG3 }\end{array}$ & $\begin{array}{c}\text { NO/YES } \\
\text { NS } \\
p<0.05 \\
\text { NS } \\
p<0.05 \\
\\
p<0.05 \\
p<0.05 \\
p<0.05 \\
p<0.05 \\
p<0.05\end{array}$ & $\begin{array}{l}\text { NO/YES } \\
p<0.05 \\
p<0.05 \\
p<0.05 \\
p<0.05 \\
p<0.05 \\
p<0.05 \\
p<0.05 \\
p<0.05 \\
p<0.05\end{array}$ & $\mathrm{NO} / \mathrm{NO}$ & NO/NO & $\begin{array}{l}\text { BW: } \\
\text { IG1: } \\
\text { IG2: } 7 \% \\
\text { IG3: } 8 \% \\
\text { IG4: } \sim 10 \% \\
\text { B 10: } \\
\text { IG1: } \sim 7 \% \\
\text { IG2: } 10 \% \\
\text { IG3: 9\% } \\
\text { IG4: 12\% }\end{array}$ \\
\hline
\end{tabular}


Table 3. Cont.

\begin{tabular}{|c|c|c|c|c|c|c|c|c|c|c|c|c|}
\hline \multirow{2}{*}{$\begin{array}{c}\text { Author(s) } \\
\text { [36-62] }\end{array}$} & \multirow[t]{2}{*}{ Location } & \multirow{2}{*}{$\begin{array}{l}\text { Study } \\
\text { Design }\end{array}$} & \multirow{2}{*}{$\begin{array}{l}\text { MS Diagnosis } \\
\text { Criteria [1] }\end{array}$} & \multirow{2}{*}{$\begin{array}{l}\text { Sample/Groups/ } \\
\text { Characteristics Studied }\end{array}$} & \multirow{2}{*}{$\begin{array}{l}\text { Duration } \\
\text { (months) }\end{array}$} & \multirow{2}{*}{$\begin{array}{c}\text { Body Composition } \\
\text { Measurement Instrument }\end{array}$} & \multirow{2}{*}{$\begin{array}{c}\text { Intervention } \\
\text { Method/Comparative Statistical } \\
\text { Analysis of the BC }\end{array}$} & \multicolumn{4}{|c|}{$\begin{array}{c}\text { Anthropometric Parameters and Measurement Unit } \\
\text { Analyzed (Statistical results) }\end{array}$} & \multirow{2}{*}{$\begin{array}{c}\text { Decreases in Body Composition } \\
\text { Mean } \pm \text { SD or Mean } \pm \text { (SE) or } \\
\text { Mean (CI, } 95 \%)\end{array}$} \\
\hline & & & & & & & & BW (kg/\%) & BF (kg or \%) & $\begin{array}{c}\mathrm{BMI} \\
\left(\mathrm{kg} / \mathrm{m}^{2} / \%\right)\end{array}$ & $\begin{array}{c}\mathrm{WC} \\
(\mathrm{cm} / \%)\end{array}$ & \\
\hline [40] & $\begin{array}{l}\text { Europe } \\
\text { (Austria) }\end{array}$ & RCT & ATPIII & $\begin{array}{c}\mathrm{n}=71 \\
\text { IG1: } 36 \\
\text { IG2: } 35 \\
\text { Men: } 100 \% \\
\text { Age: } 36-66\end{array}$ & 0.75 & $\mathrm{NE}$ & $\begin{array}{l}\text { Diet, exercise } \\
\text {-Within groups: } \\
\text { IG1 (moderate altitude exercise) } \\
\text { IG2 (sea level exercise) } \\
\text { - Between groups }\end{array}$ & $\begin{array}{l}\text { YES/NO } \\
p<0.001 \\
p<0.001 \\
\text { NS }\end{array}$ & $\begin{array}{c}\mathrm{YES} / \mathrm{NO} \\
\begin{array}{c}p<0.001 \\
p<0.001\end{array} \\
\mathrm{NE}\end{array}$ & $\begin{array}{l}\mathrm{YES} / \mathrm{NO} \\
\begin{array}{c}p<0.001 \\
p<0.001\end{array} \\
\mathrm{NS}\end{array}$ & $\begin{array}{c}\mathrm{YES} / \mathrm{NO} \\
\begin{array}{c}p<0.001 \\
p<0.001\end{array} \\
\mathrm{NE}\end{array}$ & $\begin{array}{c}\text { BW: } \\
\text { IG1: } 3.22 \pm 1.91 \mathrm{~kg} \\
\text { IG2: } 3.04 \pm 2.16 \mathrm{~kg} \\
\text { BF: NE } \\
\text { BMI: } \\
\text { IG1: } 0.81 \mathrm{~kg} / \mathrm{m}^{2} \\
\text { IG2: } 0.69 \mathrm{~kg} / \mathrm{m}^{2} \\
\text { WC: } \mathrm{NE}\end{array}$ \\
\hline [41] & $\begin{array}{l}\text { Europe } \\
\text { (France) }\end{array}$ & RCT & OMS & $\begin{array}{c}\mathrm{n}=78 \\
\text { IG1: } 24 \\
\text { IG2: } 24 \\
\text { IG3: } 30 \\
\text { Women: } 56 \% \\
\text { Age: } 50-70\end{array}$ & 12 & $\begin{array}{l}\text { DXA (Hologic QDR } 4500 \\
\text { series; Waltham, USA) }\end{array}$ & $\begin{array}{l}\text { Diet, exercise } \\
\text { - Within groups: IG1 } \\
\text { (moderate-resistance and } \\
\text { moderate-endurance) IG2 } \\
\text { (high-resistance and } \\
\text { moderate-endurance) IG3 } \\
\text { (moderate-resistance and } \\
\text { high-endurance) } \\
\text { - Between groups }\end{array}$ & $\begin{array}{l}\text { NO/YES } \\
p<0.001 \\
p<0.001 \\
p<0.001 \\
\text { NS }\end{array}$ & $\begin{array}{c}\text { NO/YES } \\
p<0.001 \\
p<0.001 \\
p<0.001 \\
\text { NS }\end{array}$ & $\mathrm{NO} / \mathrm{NO}$ & $\begin{array}{c}\text { NO/YES } \\
p<0.001 \\
p<0.001 \\
p<0.001 \\
\text { NS }\end{array}$ & $\begin{array}{c}\text { BW: } \\
\text { All: } 6.3 \pm 7.2 \% \\
\text { IG1: } 5.9 \pm 5.8 \% \\
\text { IG2: } 8.4 \pm 8.9 \% \\
\text { IG3: } 4.7 \pm 6.7 \% \\
\text { BF: } \\
\text { All: } 1.7 \pm 1.7 \% \\
\text { IG1: } 1.8 \pm 1.5 \% \\
\text { IG2: } 2.1 \pm 2.3 \% \\
\text { IG3: } 1.3 \pm 1.3 \% \\
\text { WC: } \\
\text { All: } 7.7 \pm 6.2 \% \\
\text { IG1 } 7.7 .7 \pm 6.6 \% \\
\text { IG2: } 9.5 \pm 6.8 \% \\
\text { IG3: } 6.3 \pm 5 \% \\
\end{array}$ \\
\hline [42] & $\begin{array}{l}\text { Europe } \\
\text { (Spain) }\end{array}$ & RCT & ATPIII & $\begin{array}{l}\mathrm{n}=40 \\
\text { IG1: } 20 \\
\text { IG2: } 20 \\
\text { Women: } 67 \% \\
\text { Age: } 50-66\end{array}$ & 3 & $\mathrm{NE}$ & $\begin{array}{l}\text { Diet, exercise } \\
\text { - Within groups: } \\
\text { IG1 } \\
\text { IG2 (exercise) } \\
\text { - Between groups }\end{array}$ & $\begin{array}{l}p<0.05 \\
p<0.05 \\
p<0.05\end{array}$ & $\begin{array}{l}p<0.05 \\
p<0.05 \\
p<0.05\end{array}$ & $\begin{array}{l}p<0.05 \\
p<0.05 \\
p<0.05\end{array}$ & $\begin{array}{c}p<0.05 \\
p<0.05 \\
\text { NS }\end{array}$ & $\begin{array}{c}\text { BW: } \\
\text { IG1: } 5.38 \mathrm{~kg} \\
\text { IG1: } 6.23 \pm(0.83) \% \\
\text { IG2: } 8.38 \mathrm{~kg} \\
\text { IG2: } 8.45 \pm(0.76) \% \\
\text { BF: } \\
\text { IG1: } 2.76 \% \\
\text { IG2: } 4.5 \% \\
\text { BMI: } \\
\text { IG1: } 2.09 \mathrm{~kg} / \mathrm{m}^{2} \\
\text { IG2: } 3.26 \mathrm{~kg} / \mathrm{m}^{2} \\
\text { WC: } \\
\text { IG1: } 3.72 \mathrm{~cm} \\
\text { IG2: } 4.18 \mathrm{~cm}\end{array}$ \\
\hline [43] & $\begin{array}{l}\text { North } \\
\text { America } \\
\text { (USA) }\end{array}$ & RCT & ATP III & $\begin{array}{c}\mathrm{n}=21 \\
\text { IG1: } 11 \\
\text { IG2: } 10 \\
\text { Men: } 52 \% \\
\text { Age: } 66.2 \pm 1.1\end{array}$ & 3 & $\begin{array}{l}\text { Dual-x-ray absorptiometry } \\
\text { (DEXA; Lunar Prodigy, } \\
\text { Madison, WI); Height was } \\
\text { measured with a } \\
\text { wall-mounted stadiometer } \\
\text { and weight was recorded on } \\
\text { a digital scale in a } \\
\text { hospital gown }\end{array}$ & $\begin{array}{l}\text { Diet, exercise } \\
\text { - Within ngroups: } \\
\text { IG1 (high glycemic diet) } \\
\text { IG2 (low glycemic diet) } \\
\text { - Between groups }\end{array}$ & $\begin{array}{c}p<0.001 \\
p<0.001 \\
\quad \text { NS }\end{array}$ & $\begin{array}{l}p<0.001 \\
p<0.001 \\
\quad \text { NS }\end{array}$ & $\begin{array}{l}p<0.001 \\
p<0.001 \\
\quad \text { NS }\end{array}$ & $\begin{array}{l}p<0.05 \\
p<0.05 \\
p<0.05\end{array}$ & $\begin{array}{c}\text { BW: } \\
\text { IG1: } 11 \mathrm{~kg} \\
\text { IG2: } 6.8 \mathrm{~kg} \\
\text { BF: } \\
\text { IG1: } 8.9 \mathrm{~kg} \\
\text { IG2: } 5.7 \mathrm{~kg} \\
\text { BMI: } \\
\text { IG1: } 3.6 \mathrm{~kg} / \mathrm{m}^{2} \\
\text { IG2: } 2.7 \mathrm{~kg} / \mathrm{m}^{2} \\
\text { WC: } \\
\text { IG1: 10\% } \\
\text { IG2: } \sim 6 \%\end{array}$ \\
\hline
\end{tabular}


Table 3. Cont.

\begin{tabular}{|c|c|c|c|c|c|c|c|c|c|c|c|c|}
\hline \multirow{2}{*}{$\begin{array}{l}\text { Author(s) } \\
{[36-62]}\end{array}$} & \multirow[t]{2}{*}{ Location } & \multirow{2}{*}{$\begin{array}{l}\text { Study } \\
\text { Design }\end{array}$} & \multirow{2}{*}{$\begin{array}{l}\text { MS Diagnosis } \\
\text { Criteria [1] }\end{array}$} & \multirow{2}{*}{$\begin{array}{l}\text { Sample/Groups/ } \\
\text { Characteristics Studied }\end{array}$} & \multirow{2}{*}{$\begin{array}{l}\text { Duration } \\
\text { (months) }\end{array}$} & \multirow{2}{*}{$\begin{array}{l}\text { Body Composition } \\
\text { Measurement Instrument }\end{array}$} & \multirow{2}{*}{$\begin{array}{l}\text { Intervention } \\
\text { Method/Comparative Statistical } \\
\text { Analysis of the BC }\end{array}$} & \multicolumn{4}{|c|}{$\begin{array}{c}\text { Anthropometric Parameters and Measurement Unit } \\
\text { Analyzed (Statistical results) }\end{array}$} & \multirow{2}{*}{$\begin{array}{c}\text { Decreases in Body Composition } \\
\text { Meaa } \pm \text { SD or Mean } \pm \text { (SE) or } \\
\text { Mean (CI, } 95 \%)\end{array}$} \\
\hline & & & & & & & & BW (kg/\%) & BF (kg or \%) & $\begin{array}{c}\text { BMI } \\
\left(\mathrm{kg} / \mathrm{m}^{2} / \%\right)\end{array}$ & $\begin{array}{c}\mathrm{WC} \\
(\mathrm{cm} / \%)\end{array}$ & \\
\hline [44] & $\begin{array}{l}\text { Oceania } \\
\text { (Australia) }\end{array}$ & RCT & IDF & $\begin{array}{c}\mathrm{n}=58 \\
\text { IG1: } 20 \\
\text { IG2: } 19 \\
\text { CG: } 19 \\
\text { Men: } 59 \% \\
\text { Age: } 55 \pm 6\end{array}$ & 3 & $\begin{array}{l}\text { Dual-energy X-ray } \\
\text { absorptiometry (DXA, } \\
\text { GE-LUNAR Prodigy } \\
\text { Advance PA+130510, GE } \\
\text { Medical Systems, Lunar, } \\
\text { Madison, WI, USA) }\end{array}$ & $\begin{array}{l}\text { Diet, exercise } \\
\text { - Within groups: } \\
\text { IG1 (diet) } \\
\text { IG2 (diet and exercise) } \\
\text { - Between groups: } \\
\text { IG1 vs. IG2 } \\
\text { IG1 vs. CG } \\
\text { IG2 vs. CG }\end{array}$ & $\begin{array}{l}p<0.001 \\
p<0.001 \\
\\
\quad \text { NS } \\
p<0.01 \\
p<0.01\end{array}$ & $\begin{array}{l}p<0.001 \\
p<0.001 \\
\\
\quad \text { NS } \\
p<0.01 \\
p<0.01\end{array}$ & $\begin{array}{l}p<0.001 \\
p<0.001 \\
\\
\quad \text { NS } \\
p<0.01 \\
p<0.01\end{array}$ & $\begin{array}{l}p<0.001 \\
p<0.001 \\
\\
\\
\quad \text { NS } \\
p<0.05 \\
p<0.01 \\
p<0.01\end{array}$ & $\begin{array}{c}\text { BW: } \\
\text { IG1: } 7.1 \pm 2.9 \mathrm{~kg} \\
\text { IG2: } 8.7 \pm 4.6 \mathrm{~kg} \\
\text { BF: } \\
\text { IG1: } 5.2 \pm 3.0 \mathrm{~kg} \\
\text { IG2: } 7.0 \pm 3.9 \mathrm{~kg} \\
\text { BMI: } \\
\text { IG1: } 2.4 \pm 1.0 \mathrm{~kg} / \mathrm{m}^{2} \\
\text { IG2: } 2.9 \pm 1.4 \mathrm{~kg} / \mathrm{m}^{2} \\
\text { WC: } \\
\text { IG1: } 6.7 \pm 3.2 \mathrm{~cm} \\
\text { IG2: } 10.0 \pm 5.2 \mathrm{~cm}\end{array}$ \\
\hline [45] & $\begin{array}{l}\text { North } \\
\text { Ammerica } \\
\text { (USA) }\end{array}$ & $\begin{array}{c}\mathrm{RCT} \\
\text { (parallel-arm) }\end{array}$ & ATPIII & $\begin{array}{l}n=32 \\
\text { IG1: } 8 \\
\text { IG2: } 9 \\
\text { III3: } 8 \\
\text { IG4: } 7 \\
\text { Men: } 100 \% \\
\text { Age: } 59 \pm 7\end{array}$ & 3 & $\begin{array}{l}\text { Tanita BC-418 Segmental } \\
\text { Body Composition } \\
\text { Analyzer/Scale (Tanita Inc. } \\
\text { Tokyo, Japan), which has } \\
\text { been shown to correlate } \\
\text { strongly }(r \geq 0.95, p<0.001) \\
\text { with both whole-body and } \\
\text { regional composition values } \\
\text { obtained using the gold } \\
\text { standard, dual-energy } X \text {-ray } \\
\text { absorptiometry (DEXA) }\end{array}$ & $\begin{array}{l}\text { Diet, exercise } \\
\text { - Within groups: } \\
\text { IG1 (low fat diet) } \\
\text { IG2 (low fat diet and exercise) } \\
\text { IG3 (carbohydrate-restricted diet) } \\
\text { IG4 (carbohydrate-restricted diet } \\
\text { and exercise) } \\
\text {-Between groups }\end{array}$ & $\mathrm{NO} / \mathrm{NO}$ & $\mathrm{NO} / \mathrm{NO}$ & NO/NO & $\begin{array}{c}\text { YES/NO } \\
p<0.01 \\
p<0.01 \\
p<0.01 \\
p<0.01 \\
\text { NS }\end{array}$ & $\begin{array}{l}\text { WC: } \\
\text { IG1: } 5 \mathrm{~cm} \\
\text { IG2: } 10 \mathrm{~cm} \\
\text { IIG3: } 8 \mathrm{~cm} \\
\text { IG4: } 10 \mathrm{~cm}\end{array}$ \\
\hline [46] & $\begin{array}{l}\text { South } \\
\text { America } \\
\text { (Brazil) }\end{array}$ & RCT & ATPIII & $\begin{array}{c}\mathrm{n}=75 \\
\text { IG1: } 25 \\
\text { IG2: } 25 \\
\text { CG: } 25 \\
\text { Men: } 65 \% \\
\text { Age: } 30-55\end{array}$ & 12 & $\begin{array}{l}\text { Bioelectrical impedance } \\
\text { (Omron HBF } 306 \\
\text { Bioimpedance Analyzer) and } \\
\text { WC was measured between } \\
\text { the last rib and the iliac crest }\end{array}$ & $\begin{array}{l}\text { Diet, exercise } \\
\text { CG (high CH diet and exercise } \\
\text { recommendations) } \\
\text { - Within groups: } \\
\text { II1 (low CH diet and walking) } \\
\text { IG2 (low CH diet and aerobic } \\
\text { exercise) } \\
\text {-Between groups }\end{array}$ & $\begin{array}{l}p<0.001 \\
p<0.001\end{array}$ & $\begin{array}{l}p<0.001 \\
p<0.001\end{array}$ & $\begin{array}{l}p<0.001 \\
p<0.001\end{array}$ & $\begin{array}{l}p<0.001 \\
p<0.001\end{array}$ & $\begin{array}{c}\text { BW: } \\
\text { IG1: } 9 \mathrm{~kg} \\
\text { IG2: } 11 \mathrm{~kg} \\
\text { CG: } 8 \mathrm{~kg} \\
\text { BF: } \\
\text { IG1: } 3 \% \\
\text { IG2: } 3 \% \\
\text { CG: } 2 \% \\
\text { BMI: } \\
\text { IG1 } 2.9 \mathrm{~kg} / \mathrm{m}^{2} \\
\text { IG:2: } 3.5 \mathrm{~kg} / \mathrm{m}^{2} \\
\text { CG: } 2.9 \mathrm{~kg} / \mathrm{m}^{2} \\
\text { WC: } \\
\text { IG1: } 14 \mathrm{~cm} \\
\text { IG2: } 14 \mathrm{~cm} \\
\text { CG: } 14 \mathrm{~cm}\end{array}$ \\
\hline
\end{tabular}


Table 3. Cont.

\begin{tabular}{|c|c|c|c|c|c|c|c|c|c|c|c|c|}
\hline \multirow{2}{*}{$\begin{array}{c}\text { Author(s) } \\
\text { [36-62] }\end{array}$} & \multirow[t]{2}{*}{ Location } & \multirow{2}{*}{$\begin{array}{l}\text { Study } \\
\text { Design }\end{array}$} & \multirow{2}{*}{$\begin{array}{l}\text { MS Diagnosis } \\
\text { Criteria [1] }\end{array}$} & \multirow{2}{*}{$\begin{array}{c}\text { Sample/Groups/ } \\
\text { Characteristics Studied }\end{array}$} & \multirow{2}{*}{$\begin{array}{l}\text { Duration } \\
\text { (months) }\end{array}$} & \multirow{2}{*}{$\begin{array}{c}\text { Body Composition } \\
\text { Measurement Instrument }\end{array}$} & \multirow{2}{*}{$\begin{array}{c}\text { Intervention } \\
\text { Method/Comparative Statistical } \\
\text { Analysis of the BC }\end{array}$} & \multicolumn{4}{|c|}{$\begin{array}{c}\text { Anthropometric Parameters and Measurement Unit } \\
\text { Analyzed (Statistical results) }\end{array}$} & \multirow{2}{*}{$\begin{array}{c}\text { Decreases in Body Compositior } \\
\text { Mean } \pm \text { SD or Mean } \pm \text { (SE) or } \\
\text { Mean (CI, 95\%) }\end{array}$} \\
\hline & & & & & & & & $\mathrm{BW}(\mathrm{kg} / \%)$ & $\mathrm{BF}$ (kg or \%) & $\underset{\left(\mathrm{kg} / \mathrm{m}^{2} / \%\right)}{\mathrm{BMI}}$ & $\begin{array}{c}\mathrm{WC} \\
(\mathrm{cm} / \%)\end{array}$ & \\
\hline [47] & $\begin{array}{c}\text { Oceania } \\
\text { (Australia) }\end{array}$ & $\begin{array}{c}\mathrm{RCT} \\
\text { (parallel } \\
\text { group) }\end{array}$ & ATPIII & $\begin{array}{c}\mathrm{n}=38 \\
\text { IG1: } 13 \\
\text { IG2: } 13 \\
\text { CG: } 12 \\
\text { Women: } 100 \% \\
\text { Age: } 55 \pm 1\end{array}$ & 3 & $\begin{array}{l}\text { DEXA scan (GE-LUNAR } \\
\text { Prodigy Advance } \\
\text { PAp130510; GE Medical } \\
\text { Systems, Lunar, Madison, } \\
\text { Wisconsin, USA); BW, using } \\
\text { a digital scale. WC at the } \\
\text { midpoint between the lowest } \\
\text { rib and iliac crest, and hip } \\
\text { circumference at the level of } \\
\text { the greater trochanters }\end{array}$ & $\begin{array}{l}\text { Diet, exercise } \\
\text { - Within groups: } \\
\text { IG1 (diet) } \\
\text { IG2 (diet and exercise) } \\
\text { - Between groups: } \\
\text { IG1 vs. II2 } \\
\text { IG1 vs. CG } \\
\text { IG2 vs. CG }\end{array}$ & $\begin{array}{l}p<0.05 \\
p<0.01 \\
p<0.01\end{array}$ & $\begin{array}{l}p<0.005 \\
p<0.001 \\
p<0.001\end{array}$ & $\begin{array}{l}\text { NS } \\
p<0.01 \\
p<0.00\end{array}$ & $\begin{array}{l}p<0.01 \\
p<0.001 \\
p<0.001\end{array}$ & $\begin{array}{c}\text { BW: } \\
\text { IG1: } 7.9 \pm(0.8) \mathrm{kg} \\
\text { IG: } 8.2 \pm(0.8) \% \\
\text { IG2: } 10.4 \pm(1.1) \mathrm{kgIG}: 10.7 \pm \\
(0.8) \% \\
\text { BF: } \\
\text { IG1: } 5.7 \pm(0.9) \mathrm{kg} \\
\text { IG2: } 8.5 \pm(1.0) \mathrm{kg} \\
\text { BMII } \\
\text { IG1: } 2.7 \pm(0.3) \mathrm{kg} / \mathrm{m}^{2} \\
\text { IG2: } 3.4 \pm(0.3) \mathrm{kg} / \mathrm{m}^{2} \\
\text { WC: II } 1: 7.0 \pm(0.8) \mathrm{cm} \\
\text { IG2: } 10.9 \pm(1.2) \mathrm{cm}\end{array}$ \\
\hline [48] & $\begin{array}{l}\text { North } \\
\text { America } \\
\text { (USA) }\end{array}$ & RCT & ATPIII & $\begin{array}{l}\mathrm{n}=24 \\
\text { IG1: } 12 \\
\text { IG2: } 12 \\
\text { Women: } 83 \% \\
\text { Age: } 25-80\end{array}$ & 3 & $\mathrm{NE}$ & $\begin{array}{l}\text { Diet, exercise } \\
\text { - Within groups: } \\
\text { IG1 (without interest, for the use } \\
\text { of supplementation) } \\
\text { IG2 (Mediterranean diet, exercise) } \\
\text { - Between groups }\end{array}$ & $\begin{array}{l}\text { NO/YES } \\
p<0.01 \\
p<0.01 \\
\text { NS }\end{array}$ & NO/NO & $\begin{array}{l}\text { YES/YES } \\
p<0.01 \\
p<0.01 \\
\text { NS }\end{array}$ & $\begin{array}{l}p<0.01 \\
p<0.01 \\
\text { NS }\end{array}$ & $\begin{array}{c}\text { BW: } \\
\text { IG1: } 6.8 \pm \pm(1.1) \% \\
\text { IG2: } 5.2 \pm(1.1) \% \text { BMI: } \\
\text { IG1:2.1 } \pm(0.3) \mathrm{kg} / \mathrm{m}^{2} \\
\text { IG1: } 6.7 \pm(0.9) \% \\
\text { IG2: } 1.8 \pm(1.1) \mathrm{kg} / \mathrm{m}^{2} \\
\text { IG2: } 5.2 \pm(1.1) \% \\
\text { WC: } \\
\text { IG1: } 5.9 \pm(1.1) \% \\
\text { IG2: } 5.4 \pm(1.1) \%\end{array}$ \\
\hline [49] & $\begin{array}{c}\text { Oceania } \\
\text { (Australia) }\end{array}$ & RCT & ATPIII & $\begin{array}{c}\mathrm{n}=59 \\
\text { IG1: } 200 \\
\text { IG2: } 20 \\
\text { CG: } 19 \\
\text { Men: } 59 \% \\
\text { Age: } 55 \pm 1\end{array}$ & 3 & $\begin{array}{l}\text { Dual-energy X-ray } \\
\text { absorptiometry scan } \\
\text { (GE-LUNAR Prodigy } \\
\text { Advance PA+130510; GE } \\
\text { Medical Systems, Lunar, } \\
\text { Madison, WI); BW was } \\
\text { measured using a digital } \\
\text { scale and WC was measured } \\
\text { at the midpoint between the } \\
\text { lowest rib and iliac crest and } \\
\text { hip circumference at the } \\
\text { level of the greater } \\
\text { trochanters }\end{array}$ & $\begin{array}{l}\text { Diet, exercise } \\
\text { - Within groups: } \\
\text { IG1 (diet) } \\
\text { IG2 (diet and exercise) } \\
\text { - Between groups: } \\
\text { IG1 vs. II 2 } \\
\text { IG vs. CG } \\
\text { IG2 vs. CG }\end{array}$ & $\begin{array}{c}p<0.001 / \mathrm{NE} \\
p<0.001 / \mathrm{NE} \\
\\
\mathrm{NS} / \mathrm{NS} \\
p<0.01 / \mathrm{NE} \\
p<0.01 / \mathrm{NE}\end{array}$ & $\begin{array}{l}p<0.001 \\
p<0.001 \\
\quad \text { NS } \\
p<0.01 \\
p<0.01\end{array}$ & $\begin{array}{l}p<0.001 \\
p<0.001 \\
\quad \text { NS } \\
p<0.01 \\
p<0.01\end{array}$ & $\begin{array}{l}p<0.001 \\
p<0.001 \\
p<0.01 \\
p<0.01 \\
p<0.01\end{array}$ & $\begin{array}{c}\text { BW: } \\
\text { IG1: } 7.1 \pm(0.6) \mathrm{kg} \\
\text { IG1: } 7.6 \pm(0.7) \% \\
\text { IG2: } 8.4 \pm(0.1) \mathrm{kg} \\
\text { IG2: } 8.7 \pm(0.9) \% \\
\text { BF: } \\
\text { IG1:5.2 } \pm(0.7) \mathrm{kg} \\
\text { IG2: } 6.9 \pm(0.9) \mathrm{kg} \\
\text { BMI: } \\
\text { IG1:2.4 } \\
\text { IG2: } 2.8 \pm(0.2) \mathrm{kg} / \mathrm{m}^{2} \\
\text { WC: } \\
\text { IGg/ } \\
\text { IG1: } 6.7 \pm(0.7) \mathrm{cm} \\
\text { IG2: } 9.8 \pm(1.2) \mathrm{cm}\end{array}$ \\
\hline [50] & $\begin{array}{l}\text { Oceania } \\
\text { (Australia) }\end{array}$ & RCT & ATPIII & $\begin{array}{c}\mathrm{n}=34 \\
\text { IG1: } 15 \\
\text { IG2: } 19 \\
\text { Men: } 62 \% \\
\text { Age: } 55 \pm 1\end{array}$ & 3 & $\mathrm{NE}$ & $\begin{array}{l}\text { Diet, exercise } \\
\text {-Within groups: } \\
\text { IG1 (exercise) } \\
\text { II2 } \\
\text { - Between groups }\end{array}$ & $\begin{array}{c}p<0.001 \\
p<0.001 \\
\quad \text { NS }\end{array}$ & $\begin{array}{l}p<0.001 \\
p<0.001 \\
\quad \text { NS }\end{array}$ & $\mathrm{NO} / \mathrm{NO}$ & $\begin{array}{l}p<0.001 \\
p<0.001 \\
\quad \text { NS }\end{array}$ & $\begin{array}{c}\text { BW: } \\
\text { All: } 8.2 \pm(0.7) \mathrm{kg} \\
\text { IG1: } 8.4 \pm(1.1) \mathrm{kg} \\
\text { IG2: } 8.1 \pm(0.9) \mathrm{kg} \\
\text { BF: } \\
\text { All: } 6.4 \pm(0.6) \mathrm{kg} \\
\text { IG1: } 6.9 \pm(1.1) \mathrm{kg} \\
\text { IG2: } 6.0 \pm(0.7) \mathrm{kg} \\
\text { WC: } \\
\text { All: } 8.6 \pm(0.8) \mathrm{cm} \\
\text { IG1: } 9.8 \pm(1.3 \mathrm{~cm} \\
\text { IG2: } 7.6 \pm(0.9) \mathrm{cm}\end{array}$ \\
\hline
\end{tabular}


Table 3. Cont

\begin{tabular}{|c|c|c|c|c|c|c|c|c|c|c|c|c|}
\hline \multirow{2}{*}{$\begin{array}{l}\text { Author(s) } \\
\text { [36-62] }\end{array}$} & \multirow[t]{2}{*}{ Location } & \multirow{2}{*}{$\begin{array}{l}\text { Study } \\
\text { Design }\end{array}$} & \multirow{2}{*}{$\begin{array}{l}\text { MS Diagnosis } \\
\text { Criteria [1] }\end{array}$} & \multirow{2}{*}{$\begin{array}{l}\text { Sample/Groups/ } \\
\text { Characteristics Studied }\end{array}$} & \multirow{2}{*}{$\begin{array}{l}\text { Duration } \\
\text { (months) }\end{array}$} & \multirow{2}{*}{$\begin{array}{l}\text { Body Composition } \\
\text { Measurement Instrument }\end{array}$} & \multirow{2}{*}{$\begin{array}{l}\text { Intervention } \\
\text { Method/Comparative Statistical } \\
\text { Analysis of the BC }\end{array}$} & \multicolumn{4}{|c|}{$\begin{array}{c}\text { Anthropometric Parameters and Measurement Unit } \\
\text { Analyzed (Statistical results) }\end{array}$} & \multirow{2}{*}{$\begin{array}{c}\text { Decreases in Body Composition } \\
\text { Mean } \pm \text { SD or Mean } \pm \text { (SE) or } \\
\text { Mean (CI, 95\%) }\end{array}$} \\
\hline & & & & & & & & BW (kg/\%) & BF (kg or \%) & $\begin{array}{c}\text { BMI } \\
\left(\mathrm{kg} / \mathrm{m}^{2} / \%\right)\end{array}$ & $\begin{array}{c}\mathrm{WC} \\
(\mathrm{cm} / \%)\end{array}$ & \\
\hline [51] & $\begin{array}{l}\text { North } \\
\text { America } \\
\text { (USA) }\end{array}$ & RCT & ATPIII & $\begin{array}{c}\mathrm{n}=24 \\
\text { IG1: } 12 \\
\text { IG2: } 12 \\
\text { Women } 62.5 \% \\
\text { Age: } 65.5 \pm 5.0\end{array}$ & 3 & $\begin{array}{l}\text { Hydrostatic weighing, and } \\
\text { fat mass and fat-free mass } \\
\text { were estimated using the } \\
\text { equation of Siri }\end{array}$ & $\begin{array}{l}\text { Diet, exercise } \\
\text {-Within groups: } \\
\text { IG1 } \\
\text { IG2 (diet) } \\
\text { - Between groups }\end{array}$ & $\begin{array}{l}p<0.001 \\
p<0.001 \\
p<0.05\end{array}$ & $\begin{array}{c}p<0.001 \\
p<0.001 \\
\text { NS }\end{array}$ & $\begin{array}{l}p<0.001 \\
p<0.001 \\
p<0.05\end{array}$ & $\begin{array}{c}p<0.001 \\
p<0.001 \\
\text { NS }\end{array}$ & $\begin{array}{c}\text { BW: } \\
\text { IG1: } 3.7 \pm 3.4 \mathrm{~kg} \\
\text { IG2: } 6.8 \pm 2.7 \mathrm{~kg} \\
\text { BF: } \\
\text { IG1: } 6.0 \mathrm{~kg} \\
\text { IG: } 4.5 \mathrm{~kg} \\
\text { BMI: } \\
\text { IG1: } 1.3 \mathrm{~kg} / \mathrm{m}^{2} \\
\text { IG2: } 2.4 \mathrm{~kg} / \mathrm{m}^{2} \\
\text { WC: } \\
\text { IG1: } 5.6 \mathrm{~cm} \\
\text { IG2: } 6.5 \mathrm{~cm}\end{array}$ \\
\hline [52] & $\begin{array}{l}\text { North } \\
\text { America } \\
\text { (USA) }\end{array}$ & $\begin{array}{c}\mathrm{RCT} \\
\text { (parallel-arm) }\end{array}$ & ATPIII & $\begin{array}{c}\mathrm{n}=47 \\
\text { IG1: } 24 \\
\text { IG2: } 23 \\
\text { Men: } 50 \% \\
\text { Age: } 20-65\end{array}$ & 3 & $\begin{array}{l}\text { DXA (QDR-4500W; Hologic } \\
\text { Corp, Waltham, MA); BW by } \\
\text { electronic scale (model CN20; } \\
\text { Cardinal/Detecto, Webb City, } \\
\text { MO); WC was measured } \\
\text { according to guidelines of } \\
\text { the National Heart, Lung, } \\
\text { and Blood Institute (NHLBI) }\end{array}$ & $\begin{array}{l}\text { Diet, exercise } \\
\text {-Within groups: } \\
\text { IG1 (without refined grain diet) } \\
\text { IG2 (refined grain diet) } \\
\text { - Between groups }\end{array}$ & $\begin{array}{c}p<0.001 \\
p<0.001 \\
\text { NS }\end{array}$ & $\begin{array}{c}\text { YES }(\%) / \text { NO } \\
\begin{array}{c}p<0.001 \\
p<0.001\end{array} \\
\text { NS }\end{array}$ & $\mathrm{NO} / \mathrm{NO}$ & $\begin{array}{l}\text { YES/NO } \\
p<0.001 \\
p<0.001 \\
\text { NS }\end{array}$ & $\begin{array}{c}\text { BW: } \\
\text { IG1: }: .7 \pm 3.5 \mathrm{~kg} \\
\text { IG2: } 5.3 \pm 5.2 \mathrm{~kg} \\
\text { BF: } \\
\text { IG1: } 1.2 \pm 1.3 \% \\
\text { IG2: } 1.0 \pm 1.6 \% \\
\text { WC } \\
\text { IG1: } 2.5 \pm 3.7 \mathrm{~cm} \\
\text { IG2: } 4.7 \pm 6.4 \mathrm{~cm}\end{array}$ \\
\hline [53] & $\begin{array}{c}\text { Asia } \\
\text { (Thailand) }\end{array}$ & RCT & IDF & $\begin{array}{c}\mathrm{n}=110 \\
\text { IG1: } 52 \\
\text { IG2: } 58 \\
\text { Women: } 83 \% \\
\text { Age: } 42.5 \pm 1.1\end{array}$ & 3 & $\begin{array}{l}\text { Body composition were } \\
\text { measured using BIA } \\
\text { (TANITA }{ }^{\circledR} \text { BC- }-418 \text {, Tanita } \\
\text { corp., Tokyo, Japan). WC } \\
\text { was measured using a no } \\
\text { stretchable tape with } \\
\text { measurement taken at a } \\
\text { horizontal line midway } \\
\text { between the highest point of } \\
\text { iliac crest and the lowest ribs }\end{array}$ & $\begin{array}{l}\text { Diet, intervention in lifestyle } \\
\text { Within } \\
\text { IG1: Lifestyle intervention } \\
\text { IG2: lifestylle intervention plus } \\
\text { meal replacement } \\
\text { Between groups: }\end{array}$ & $\begin{array}{l}p<0.01 \\
p<0.01 \\
p<0.05\end{array}$ & $\begin{array}{c}p<0.01 \\
p<0.01 \\
\text { NS }\end{array}$ & $\begin{array}{l}p<0.01 \\
p<0.01 \\
p<0.05\end{array}$ & $\begin{array}{c}p<0.01 \\
p<0.01 \\
\text { NS }\end{array}$ & $\begin{array}{c}\text { BW: } \\
\text { IG1: } 1.4 \mathrm{~kg} \\
\text { IG1: } 1.53 \% \\
\text { IG2: } 2.3 \mathrm{~kg} \\
\text { IG22 } 2.86 \% \\
\text { BF: } \\
\text { IGG: } 1.05 \mathrm{~kg} \\
\text { IG1: } 0.8 \% \\
\text { IG2: } 1.58 \mathrm{~kg} \\
\text { IG2: } 1 \% \\
\text { BMIIG1: } 0.49 \mathrm{~kg} / \mathrm{m}^{2} \\
\text { IG2: } 0.94 \mathrm{~kg} / \mathrm{m}^{2} \\
\text { WC } \\
\text { IG1: } 2.5 \mathrm{~cm} \\
\text { IG2: } 3.25 \mathrm{~cm}\end{array}$ \\
\hline [54] & $\begin{array}{c}\text { Asia } \\
\text { (Iran) }\end{array}$ & $\mathrm{RCT}$ & ATPIII & $\begin{array}{c}\mathrm{n}=117 \\
\text { IG: } 64 \\
\text { CG: } 53 \\
\text { Men: } 63.3 \% \\
\text { Age: } 44.2(\text { s.d. }=10.0)\end{array}$ & 6 & $\begin{array}{l}\text { BW by a calibrated scale } \\
\text { (Seca, Hamburg, Germany } \\
\text { model } 8811021658) \text { to the } \\
\text { nearest of } 0.1 \mathrm{~kg} \text {; Height by } \\
\text { stadiometer (Seca, Hamburg, } \\
\text { Germany) to the nearest of } \\
0.1 \mathrm{~cm}\end{array}$ & $\begin{array}{l}\text { Diet, intervention in lifestyle } \\
\text { - Within groups: } \\
\text { IG (My Healthy Heart Profile } \\
\text { interactive web) } \\
\text { - Between groups }\end{array}$ & $\begin{array}{l}\text { YES/NO } \\
p<0.001 \\
p<0.05\end{array}$ & NO/NO & $\begin{array}{c}\text { YES/NO } \\
p<0.001 \\
\text { NS }\end{array}$ & $\mathrm{NO} / \mathrm{NO}$ & $\begin{array}{l}\text { BW: } \\
\text { IG: } 4 \mathrm{~kg} \\
\text { BMI: } \\
\text { IG: } 1.2 \mathrm{~kg} / \mathrm{m}^{2}\end{array}$ \\
\hline
\end{tabular}


Table 3. Cont

\begin{tabular}{|c|c|c|c|c|c|c|c|c|c|c|c|c|}
\hline \multirow{2}{*}{$\begin{array}{l}\text { Author(s) } \\
\text { [36-62] }\end{array}$} & \multirow[t]{2}{*}{ Location } & \multirow{2}{*}{$\begin{array}{l}\text { Study } \\
\text { Design }\end{array}$} & \multirow{2}{*}{$\begin{array}{c}\text { MS Diagnosis } \\
\text { Criteria [1] }\end{array}$} & \multirow{2}{*}{$\begin{array}{l}\text { Sample/Groups/ } \\
\text { Characteristics Studied }\end{array}$} & \multirow{2}{*}{$\begin{array}{l}\text { Duration } \\
\text { (months) }\end{array}$} & \multirow{2}{*}{$\begin{array}{c}\text { Body Composition } \\
\text { Measurement Instrument }\end{array}$} & \multirow{2}{*}{$\begin{array}{c}\text { Intervention } \\
\text { Method/Comparative Statistical } \\
\text { Analysis of the BC }\end{array}$} & \multicolumn{4}{|c|}{$\begin{array}{l}\text { Anthropometric Parameters and Measurement Unit } \\
\text { Analyzed (Statistical results) }\end{array}$} & \multirow{2}{*}{$\begin{array}{c}\text { Decreases in Body Composition } \\
\text { Mean } \pm \text { SD or Mean } \pm \text { (SE) or } \\
\text { Mean }(\mathrm{Cl}, 95 \%)\end{array}$} \\
\hline & & & & & & & & BW (kg/\%) & BF (kg or \%) & $\begin{array}{c}\text { BMI } \\
\left(\mathrm{kg} / \mathrm{m}^{2} / \%\right)\end{array}$ & $\begin{array}{c}\mathrm{WC} \\
(\mathrm{cm} / \%)\end{array}$ & \\
\hline [55] & $\begin{array}{l}\text { Europe } \\
\text { (Greece) }\end{array}$ & RCT & ATP III & $\begin{array}{c}\mathrm{n}=47 \\
\text { CG: } 13 \\
\text { IG1: } 16 \\
\text { IG2: } 18 \\
\text { Men: } 57 \% \\
\text { Age: } 49.0 \pm 11.8\end{array}$ & 6 & $\begin{array}{l}\text { Weight and height were } \\
\text { measured on a leveled } \\
\text { platform scale and a } \\
\text { wall-mounted stadiometer, } \\
\text { to the nearest } 0.5 \mathrm{~kg} \text { and } 0.5 \\
\mathrm{~cm} \text {; } W C \text { Cas measured in the } \\
\text { middle between the } 12^{\text {th }} \text { rib } \\
\text { and the iliac crest }\end{array}$ & $\begin{array}{l}\text { Diet, intervention in lifestyle } \\
\text { CG (usual care) } \\
\text { - Within groups: } \\
\text { IG1 (face-to-face) } \\
\text { IG2 (telephone group) } \\
\text { - Between groups: } \\
\text { IG1 vs. CG } \\
\text { IG2 vs. CG }\end{array}$ & $\mathrm{NO} / \mathrm{NO}$ & $\mathrm{NO} / \mathrm{NO}$ & $\begin{array}{l}p<0.001 \\
p<0.001 \\
p<0.001 \\
p<0.001\end{array}$ & $\begin{array}{l}\text { YES/NO } \\
p<0.001 \\
p<0.001 \\
p<0.001 \\
p<0.05\end{array}$ & $\begin{array}{c}\text { BMI: } \\
\text { CG: } 0.1 \pm 1.0 \mathrm{~kg} / \mathrm{m}^{2} \\
\text { IG1: } 1.4 \pm 1.5 \mathrm{~kg} / \mathrm{m}^{2} \\
\text { IG2: } 1.2 \pm 1.4 \mathrm{~kg} / \mathrm{m}^{2} \\
\text { WC: } \\
\text { CG: } 0.5 \pm 4.4 \mathrm{~cm} \\
\text { IG1: } 4.1 \pm 5.0 \mathrm{~cm} \\
\text { IG2: } 3.5 \pm 4.4 \mathrm{~cm}\end{array}$ \\
\hline [56] & $\begin{array}{l}\text { South } \\
\text { America } \\
\text { (Brazil) }\end{array}$ & RCT & ATP III & $\begin{array}{c}\mathrm{n}=58 \\
\text { CG: } 17 \\
\text { IG1: } 21 \\
\text { IG2: } 20 \\
\text { Women: } 55.5 \% \\
\text { Age: } 30-59\end{array}$ & 3 & $\begin{array}{l}\text { Body weight measured, } \\
\text { using a properly calibrated } \\
1060 \mathrm{~kg} \text { Cauduro scale; WC, } \\
\text { with a millimeter no } \\
\text { extensiblel long tape at the } \\
\text { abdomen's maximum } \\
\text { extension }\end{array}$ & $\begin{array}{l}\text { Diet, exercise, intervention } \\
\text { in lifestyle } \\
\text { CG: (standard intervention) } \\
\text { - Within groups: } \\
\text { IG1 (group intervention) } \\
\text { IG2 (individual intervention) } \\
\text { - Between groups: } \\
\text { IG1 vs. CG } \\
\text { IG2 vs. CG } \\
\text { IG1 vs. IG2 }\end{array}$ & $\mathrm{NO} / \mathrm{NO}$ & NO/NO & $\begin{array}{l}p<0.01 \\
p<0.05 \\
p<0.05 \\
p<0.05 \\
\quad \text { NS }\end{array}$ & $\begin{array}{l}p<0.05 \\
p<0.05 \\
p<0.05 \\
p<0.05 \\
\quad \text { NS }\end{array}$ & $\begin{array}{c}\text { BMI: } \\
\text { IG1: } 1.8 \mathrm{~kg} / \mathrm{m}^{2} \\
\text { IG2: } 1.5 \mathrm{~kg} / \mathrm{m}^{2} \\
\text { WC: } \\
\text { IG1: }: 4.4 \mathrm{~cm} \\
\text { IG2: } 5.3 \mathrm{~cm}\end{array}$ \\
\hline [57] & $\begin{array}{l}\text { Europe } \\
\text { (Spain) }\end{array}$ & RCT & IDF & $\begin{array}{l}\mathrm{n}=406 \\
\text { IG: } 230 \\
\text { CG: } 176 \\
\text { Men: } 55 \% \\
\text { Age: } 18-80\end{array}$ & 36 & $\mathrm{NE}$ & $\begin{array}{l}\text { Diet, exercise, intervention } \\
\text { in lifestyle } \\
\text { CG: } 176 \text { (healthy diet with general } \\
\text { physical activity) } \\
\text { - Within groups: } \\
\text { IG (Mediterranean diet } \\
\text { and exercise) } \\
\text { - Between groups }\end{array}$ & $\begin{array}{l}\text { NE } \\
\text { NS }\end{array}$ & $\mathrm{NO} / \mathrm{NO}$ & YES/NO & $\begin{array}{c}\mathrm{NE} \\
p<0.001\end{array}$ & $\begin{array}{l}\text { BW: } \\
\text { IG: increase } \\
\text { BMI: } \\
\text { IG: increase } \\
\text { WC: } \\
\text { IG: } 0.3 \pm 6.0 \mathrm{~cm}\end{array}$ \\
\hline [58] & $\begin{array}{l}\text { Europe } \\
\text { (Germany) }\end{array}$ & $\begin{array}{l}\mathrm{RCT} \\
\text { (paralle-group) } \\
\text { groups }\end{array}$ & IDF & $\begin{array}{l}\mathrm{n}=178 \\
\text { CG } 60 \\
\text { IG1: } 60 \\
\text { IG2: } 58 \\
\text { Men: } 57 \% \\
\text { Age: } 30-60\end{array}$ & 12 & $\mathrm{NE}$ & $\begin{array}{l}\text { Diet, exercise, intervention } \\
\text { in lifestyle } \\
\text { Within groups: } \\
\text { IG1 (monitored weekly)IG2 } \\
\text { (monitored monthly) } \\
\text { - Between groups: } \\
\text { IG1 vs. IG2 } \\
\text { IG1 vs. CG } \\
\text { IG1 vs. CG }\end{array}$ & 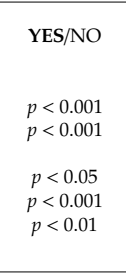 & $\mathrm{NO} / \mathrm{NO}$ & $\begin{array}{l}p<0.001 \\
p<0.001 \\
p<0.05 \\
p<0.001 \\
p<0.01\end{array}$ & $\begin{array}{l}p<0.001 \\
p<0.001 \\
p<0.05 \\
p<0.001 \\
p<0.05\end{array}$ & $\begin{array}{c}\text { BW: } \\
\text { IG1: } 12.2 \mathrm{~kg}(8.8-10.5) \\
\text { IG1: } 11.4 \%(9.8-12.9) \\
\text { IG2: } 8.8 \mathrm{~kg}(7.1-10.4) \\
\text { IG2: } 8.6 \%(7-10.2) \\
\text { BMI: } \\
\text { IG1: } 4.1 \mathrm{~kg} / \mathrm{m}^{2}(3.6-4.6) \\
\text { IG2: } 2.8 \mathrm{~kg} / \mathrm{m}^{2}(2.3-3.4) \\
\text { WC: } \\
\text { IG1: } 14.3 \mathrm{~cm}(12.3-16.3) \\
\text { IG2: } 10.8 \mathrm{~cm}(8.9-12.8) \\
\end{array}$ \\
\hline [59] & $\begin{array}{l}\text { Oceania } \\
\text { (Australia) }\end{array}$ & RCT & ATP III & $\begin{array}{c}\mathrm{n}=66 \\
\text { IG: } 31 \\
\text { CG: } 35 \\
\text { Women: } 68 \% \\
\text { Age: } 18-60\end{array}$ & 12 & $\begin{array}{l}\text { Tanita BC-418 segmental } \\
\text { body composition analyzer } \\
\text { (Tanita Corporation of } \\
\text { America Inc., Arlington } \\
\text { Heights, IL). WC was } \\
\text { measured midway between } \\
\text { the top of the iliac crest and } \\
\text { the most inferior part of the } \\
\text { rib cage }\end{array}$ & $\begin{array}{l}\text { Diet, exercise, intervention } \\
\text { in lifestyle } \\
\text { IG: without interest, for the use of } \\
\text { the gastric balloon } \\
\text { - Within groups: } \\
\text { CG (diet and exercise) }\end{array}$ & $p<0.05$ & NO/NO & $\mathrm{YES} / \mathrm{NO}$ & $\mathrm{YES} / \mathrm{NO}$ & $\begin{array}{c}\text { BW: } \\
\text { CG: } 5.3 \mathrm{~kg} \\
\text { CG: } 5.2 \% \\
\text { BMI: } \\
\text { CG: } 1.9 \mathrm{~kg} / \mathrm{m}^{2} \\
\text { WC: } \\
\text { CG: } 6.4 \mathrm{~cm}\end{array}$ \\
\hline
\end{tabular}


Table 3. Cont.

\begin{tabular}{|c|c|c|c|c|c|c|c|c|c|c|c|c|}
\hline \multirow{2}{*}{$\begin{array}{l}\text { Author(s) } \\
\text { [36-62] }\end{array}$} & \multirow[t]{2}{*}{ Location } & \multirow{2}{*}{$\begin{array}{l}\text { Study } \\
\text { Design }\end{array}$} & \multirow{2}{*}{$\begin{array}{l}\text { MS Diagnosis } \\
\text { Criteria [1] }\end{array}$} & \multirow{2}{*}{$\begin{array}{l}\text { Sample/Groups/ } \\
\text { Characteristics Studied }\end{array}$} & \multirow{2}{*}{$\begin{array}{l}\text { Duration } \\
\text { (months) }\end{array}$} & \multirow{2}{*}{$\begin{array}{c}\text { Body Composition } \\
\text { Measurement Instrument }\end{array}$} & \multirow{2}{*}{$\begin{array}{c}\text { Intervention } \\
\text { Method/Comparative Statistical } \\
\text { Analysis of the BC }\end{array}$} & \multicolumn{4}{|c|}{$\begin{array}{c}\text { Anthropometric Parameters and Measurement Unit } \\
\text { Analyzed (Statistical results) }\end{array}$} & \multirow{2}{*}{$\begin{array}{c}\text { Decreases in Body Compositio } \\
\text { Mean } \pm \text { SD or Mean } \pm \text { (SE) or } \\
\text { Mean (CI, 95\%) }\end{array}$} \\
\hline & & & & & & & & $\mathrm{BW}(\mathrm{kg} / \%)$ & BF (kg or \%) & $\begin{array}{c}\mathrm{BMI} \\
\left(\mathrm{kg} / \mathrm{m}^{2} / \%\right)\end{array}$ & $\begin{array}{c}\mathrm{WC} \\
(\mathrm{cm} / \%)\end{array}$ & \\
\hline [60] & $\begin{array}{l}\text { Europe } \\
\text { (Greece) }\end{array}$ & RCT & ATP III & $\begin{array}{c}\mathrm{n}=88 \\
\text { CG: } 29 \\
\text { IG1: } 29 \\
\text { IG2: } 30 \\
\text { Men: } 57 \% \\
\text { Age: } 49.9 \pm 10.8\end{array}$ & 6 & $\begin{array}{l}\text { BW and height were } \\
\text { measured on a levelled } \\
\text { platform scale and a } \\
\text { wall-mounted stadiometer }\end{array}$ & $\begin{array}{l}\text { Diet, exercise, intervention } \\
\text { in lifestyle } \\
\text { - Within groups: } \\
\text { IG1 (healthy food) } \\
\text { IG2 (healthy food and decrease of } \\
\text { less healthy food) } \\
\text { - Between groups: } \\
\text { II1 vs. CG } \\
\text { IG2 vs. CG }\end{array}$ & $\mathrm{NO} / \mathrm{NO}$ & $\mathrm{NO} / \mathrm{NO}$ & $\begin{array}{l}p<0.05 \\
p<0.05 \\
\\
p<0.05 \\
p<0.05\end{array}$ & $\begin{array}{l}\mathrm{YES} / \mathrm{NO} \\
p<0.05 \\
p<0.05\end{array}$ & $\begin{array}{c}\text { BMI: } \\
\text { CG: } 0.1 \pm 1.0 \mathrm{~kg} / \mathrm{m}^{2} \\
\text { IG1: } 1.2 \pm 1.4 \mathrm{~kg} / \mathrm{m}^{2} \\
\text { IG2: } 1.2 \pm 1.4 \mathrm{~kg} / \mathrm{m}^{2} \\
\text { WC: } \\
\text { CG: } 0.4 \pm 4.4 \mathrm{~cm} \\
\text { IG1: } 3.5 \pm 4.4 \mathrm{~cm} \\
\text { IG2: } 3.1 \pm 4.0 \mathrm{~cm}\end{array}$ \\
\hline [61] & $\begin{array}{l}\text { Asia } \\
\text { (South } \\
\text { Korea) }\end{array}$ & RCT & ATPIII & $\begin{array}{c}\mathrm{n}=48 \\
\text { IG: } 27 \\
\text { CG: } 21 \\
\text { Women: } 100 \% \\
\text { Age: } 62.7 \pm 9.0\end{array}$ & 12 & $\begin{array}{l}\text { BW was measured with a } \\
\text { high-precision scale (InBody } \\
220 \text {; Biospace company, } \\
\text { Seoul, Korea); WC was } \\
\text { measured midway between } \\
\text { the lowest rib and the } \\
\text { iliac crest }\end{array}$ & $\begin{array}{l}\text { Diet, exercise, intervention } \\
\text { in lifestyle } \\
\text { - Within groups: } \\
\text { IG } \\
\text { - Between groups }\end{array}$ & $\begin{array}{l}\mathrm{YES} / \mathrm{NO} \\
p<0.001 \\
p<0.001\end{array}$ & $\mathrm{NO} / \mathrm{NO}$ & $\begin{array}{l}p<0.001 \\
p<0.001\end{array}$ & $\begin{array}{l}p<0.001 \\
p<0.001\end{array}$ & $\begin{array}{c}\text { BW: } \\
\text { IG: } 4.3 \mathrm{~kg} \\
\text { BMI: } \\
\text { IG: } 1.4 \mathrm{~kg} / \mathrm{m}^{2} \\
\text { WC: } \\
\text { IG: } 9.4 \mathrm{~cm}\end{array}$ \\
\hline [62] & $\begin{array}{l}\text { Asia } \\
\text { (South } \\
\text { Korea) }\end{array}$ & RCT & ATPIII & $\begin{array}{c}\mathrm{n}=29 \\
\text { IG: } 16 \\
\text { CG: } 13 \\
\text { Women: } 100 \% \\
\text { Age: } 66.7 \pm 9.7\end{array}$ & 1 & $\begin{array}{l}\text { BW was measured with a } \\
\text { high-precision scale } \\
\text { (GM1000; Neo GMTEC, } \\
\text { Seoul, Korea); WC was } \\
\text { measured midway between } \\
\text { the lowest rib and the } \\
\text { iliac crest }\end{array}$ & $\begin{array}{l}\text { Diet, exercise, intervention } \\
\text { in lifestyle } \\
\text { - Within groups: } \\
\text { IG } \\
\text { - Between groups }\end{array}$ & $\begin{array}{l}\mathrm{YES} / \mathrm{NO} \\
p<0.001 \\
p<0.001\end{array}$ & $\mathrm{NO} / \mathrm{NO}$ & $\begin{array}{l}p<0.001 \\
p<0.001\end{array}$ & $\begin{array}{l}p<0.001 \\
p<0.001\end{array}$ & $\begin{array}{c}\text { BW: } \\
\text { IG: } 4.6 \mathrm{~kg} \\
\text { BMI: } \\
\text { IG: } 2 \mathrm{~kg} / \mathrm{m}^{2} \\
\text { WC: } \\
\text { IG: } 6.2 \mathrm{~cm}\end{array}$ \\
\hline
\end{tabular}

Body weight (BW); body fat (BF); body mass index (BMI); waist circumference (WC); control group (CG); intervention group (IG); non-significant (NS); the information is not available in the clinical trial evaluated (NE); randomized controlled trial (RCT); YES: the article includes the analysis of the parameter expressed in its correspondent units; NO: the article does not include the analysis of the parameter; carbohydrate $(\mathrm{CH})$; mono or poly-unsaturated fatty acids (MUFA, PUFA); first aerobic interval training, later diet (EXER-then-DIET); exercise and diet simultaneously (EXER + DIET); $18 \%$ proteins, mostly of vegetable origin, $55 \%$ carbohydrate and $27 \%$ fat (M-DASH); $18.4 \%$ proteins, highest proportion of animal origin, $54 \%$ and diet simultaneously (EXER + DIET); $18 \%$ proteins, mostly of vegetable origin, $55 \%$ carbohydrate and $27 \%$ fat (M-DASH); $18.4 \%$ proteins, highest proportion of animal origin, $54 \%$.
carbohydrate and $27 \%$ fat (BOLD); $27 \%$ proteins, highest proportion of animal origin, $45 \%$ carbohydrate and $27 \%$ fat (BOLD+); physical exercise performed at a moderate height of $1700 \mathrm{~m}$ (moderate altitude exercise); exercise at sea level to $200 \mathrm{~m}$ (sea level exercise) 


\section{Results}

\subsection{Search Features and Types of Interventions Identified}

A total of 2684 articles were identified, but finally only $1.6 \%$ of the overall were included on the basis on the search strategy (Figure 1). The most useful strategy search included the following variables: "metabolic syndrome" and "weight loss" or "weight reduction" or "fat loss" or "fat reduction" or "lifestyle" and "exercise" or "physical activity" or "sport" or "weightlifting"; Web of Science and PubMed are the databases that had the most clinical trials included (Table 1). After analyzing the items, they were classified into two groups according to the type of intervention used: exclusive intervention (27\%, Table 2) and multidisciplinary interventions (73\%, Table 3). The most relevant characteristics of the clinical trials included are (Tables 2 and 3): number of patients from 21 to 406 (median, 49 patients); greater proportion of mixed samples (75.7\%), 8.1\% only constituted by men and $16.2 \%$ only constituted by women; patients from 18 to 80 years old; sample groups located in Europe (35.1\%), America (29.7\%), Asia (18.9\%) and Oceania (16.2\%); Adult Treatment Panel III (ATPIII) of the National Cholesterol Education Program (NCEP), International Diabetes Federation (IDF), World Health Organization (WHO) and The Examination Committee of Criteria for "Metabolic Syndrome" in Japan as MS diagnosis criteria; temporary duration of the studies from 0.75 to 36 months (median and mode 3 months, up to 6 months, 81.1\%). The most abundant intervention modalities observed were diet, physical exercise and/or changes in lifestyle (a combination of diet and physical exercise predominates as intervention (45.9\% of the studies)).

Regarding the ten works based on exclusive interventions, the duration of the interventions in eight of them ranged from 2.5 to 6 months and just one had a duration up to 24 months. The sample size ranged from 27 to 173 patients (median, 93.5 patients). Eight of them assigned the patients into two groups (two intervention groups in five works and an intervention group together with a control group in the others three). In the two remaining works, the patients were assigned into two intervention groups and a control group. Concerning to the method of intervention, there were three works with modifications in lifestyle, four impinged changes in diet and three controlled the method of exercise.

Regarding the twenty-seven articles based on multidisciplinary interventions, the duration in the interventions ranged from 0.75 to 36 months $(77.8 \%$ with a period up to 6 months; five and one with durations of 12 and 36 months, respectively). The sample size ranged from 21 to 406 patients (median, 48 patients). Fourteen of them assigned the patients into two groups (two intervention groups in nine works and an intervention group together with a control group in the others five). Eleven works designated three groups (nine studies with two intervention groups and a control group) and finally, there were two works with four intervention groups. Concerning the methods of intervention, there were seventeen works with interventions on diet and exercise and ten with modifications in diet, exercise and lifestyle.

\subsection{The Most Significant Body Composition Changes in MS (Tables 2 and 3)}

As a response to the first secondary objective, the analysis reveals that multidisciplinary interventions report the greatest changes in body composition. In this context, Luley and co-workers show the largest changes in BW, BMI and WC [58]. This was a 12-month multidisciplinary intervention in diet, exercise and lifestyle, carried out in Europe with a sample of 178 patients (mainly men, 57\%) between 30 and 60 years of age, randomized in a control group and two intervention groups. All the subjects were advised to increase their usual daily physical activity following Magdeburg Dual Diet (500 kcal per day and low in carbohydrates, preferably with a low glycemic index). Diet and exercise were monitored in IG1 weekly by letter, in IG2 monthly by telephone, whereas CG was not monitored. The changes of each variable measured was: $12.2 \mathrm{~kg}$ (CI 95\%, 10.5-13.8), (11.4\% (9.8-12.9)), $4.1 \mathrm{~kg} / \mathrm{m}^{2}$ (3.6-4.6) (12\%) and $14.3 \mathrm{~cm}$ (12.3-16.2) (12.1\%) in IG1 for BW, BMI and WC, respectively.

The Malin and Bonfanti clinical trials, based on multidisciplinary intervention techniques, show the best results for reducing BF $[39,43]$. On the other hand, the clinical trial of Bonfanti and coworkers is 
a 3-month European study involving patients of both sexes, over 50 years old $(n=36)$ and randomized in four intervention groups [39]. In two groups, the subjects followed hypocaloric diets with different proportion in carbohydrates, protein and fats and in the remaining groups exercise was also added. Subjects in IG1 followed a Mediterranean diet while subjects in IG2 followed a low fat diet rich in complex carbohydrates. In IG3 and IG4, the respective diets of IG1 and IG2 were combined with aerobic exercise. The change of BF was $12 \%$ in IG4.

It was analyzed whether the included clinical trials that have obtained the greatest variations in body composition have developed the items recommended by the CONSORT method [63]. Between the studies here included the following three have reported the highest number of positives results: Luley et al., Malin et al. and Bonfanti et al. (59.4\%, 37.84\% and 35.2\%, respectively) [39,43,58].

As a response to the second secondary objective, energy restriction in diet was a common characteristic of the clinical trials that achieved the greatest changes in body composition in this review, occasionally combined with structured physical exercise.

\subsection{Anthropometric Parameters and Units of MS Changes in Body Composition Measurements (Tables 2 and 3)}

With regard to the articles based on exclusive interventions, all of them study and report a statistically significant $(p$-value $<0.05$ ) changes in BW within the intervention groups (final vs. baseline levels), with the exception of an intervention group in which no significant differences in BF, BMI and WC were observed [32]. Regarding to the differences in BW between intervention groups, they turned out to be statistically significant in 50\% of the total studies (Figure 2a).

In the case of $\mathrm{BF}, 50 \%$ of the works had results expressed in $\mathrm{kg}$ or percentage. Only one of them presented statistically significant differences between the analyzed groups. On the other hand, nine of the ten trials studied the changes in BMI in $\mathrm{kg} / \mathrm{m}^{2}$, obtaining statistically significant changes among groups in five of the nine studies. Finally, the loss of $\mathrm{cm}$ in WC was collected in all the works, reporting statistically significant changes in four of the ten trials.

Concerning multidisciplinary intervention trials (Figure $2 b$ ), $85.2 \%$ of them included the study of BW changes in $\mathrm{kg}$ or/and relative units. All but three of the trials studied and reported a statistically significant ( $p$-value $<0.05$ ) changes in BW ( $\mathrm{kg}$ or relative units) within the intervention groups (final vs. baseline levels), except in $[36,37,39]$. Related to the differences in BW between intervention groups, they turned out to be statistically significant $(p$-value $<0.05$ ) in $52.2 \%$ of the cases (Figure $2 b$ ).

The variable BF was not included in $44.4 \%$ of the works analyzed, neither in absolute terms $(\mathrm{kg}$ or \%) nor in relative units. Fourteen studies reported significant differences within the intervention groups but eight studied reported non-significant differences between the intervention groups.

Concerning BMI, twenty-one works included the study of the changes in absolute units and one of them incorporates their study in relative units. Among these articles, twelve had significant differences between groups. Finally, the loss in WC (in $\mathrm{cm}$ ) was collected in $88.9 \%$ of the works, registering statistically significant changes in twelve of the twenty-four trials. The study of the relative changes appeared in three studies, with statistically significant differences between intervention groups in one of them.

In order to reach the last secondary objective of this review, from all the articles selected it is possible to conclude that the most analyzed parameter was WC ( $88.9 \%$ of the trials), followed by BW (85.2\%), BMI (77.8\%) and BF (55.6\%). Regarding WC, 44.4\% of the articles reported statistically significant differences among groups, $37 \%$ reported non-significant differences and $7.4 \%$ did not specify the classification of the differences. In the case of BW, BMI and BF the percentages were $44.4 \%, 33.3 \%$ and $7.4 \%, 44.4 \%, 29.6 \%$ and $3.7 \%$ and $18.5 \%, 29.6 \%$ and $7.4 \%$, respectively (Figure $2 \mathrm{c}$ ). 

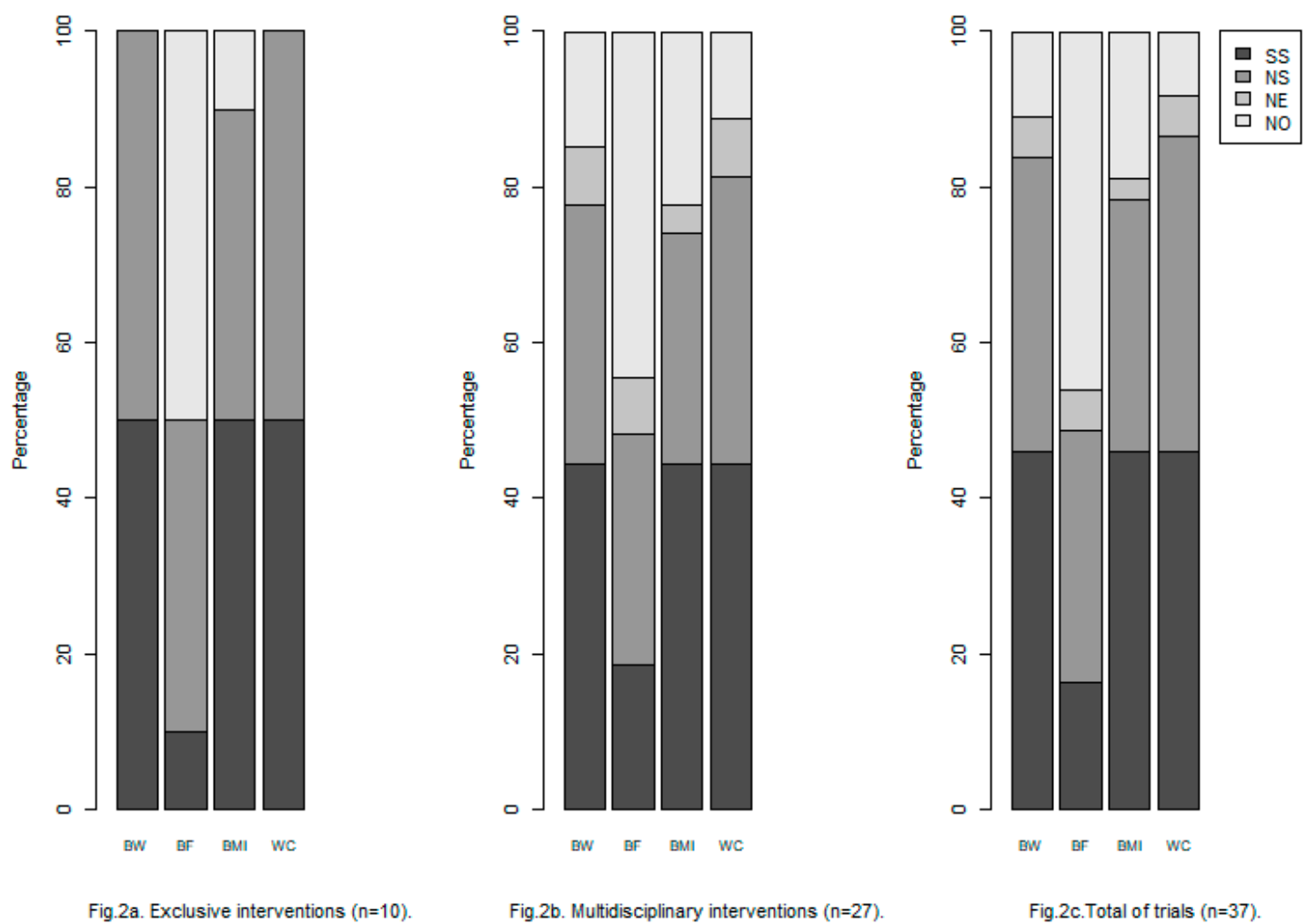

Figure 2. Percentage of articles that studied the parameter analyzed in this systematic review and results of comparison between groups. body weight (BW); body fat (BF); body mass index (BMI); control group (CG); intervention group (IG); non-significant (NS); statistically significant (SS); the information is not available in the clinical trial evaluated (NE); the article does not include its study (NO); waist circumference (WC).

\section{Discussion}

Due to the high rates of MS worldwide [5-7] and the fact that the guidelines for MS interventions are scarce and old (dietary and physical exercise interventions) [64-66], it is necessary to delve into which practical guidance would report a greater variation of body composition. In order to reach the fourth secondary objective, Tables 4 and 5 were done [67-74] (European Guidelines and institutional guides such as: AACE, ADA, ACE, AHA, NHLBI). The selected guides had to meet the following criteria: to have a degree of evidence analysis; the main objective was to cause changes in body composition; review articles would have been published in journals indexed in the Journal Citation Reports (Web of Science) between 2005 and 2018. 
Table 4. Guidelines and consensus on the treatment of overweight, obesity, type 2 diabetes mellitus and metabolic syndrome: adults ${ }^{\dagger}$.

\begin{tabular}{|c|c|c|c|}
\hline & & Author [67-74] & Recommendations in Dietary Intervention and Exercise \\
\hline \multirow{2}{*}{ 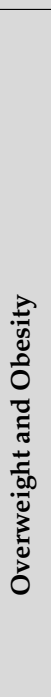 } & 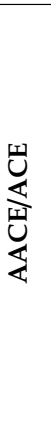 & [67] & $\begin{array}{l}\text { Dietary intervention: energy reduction }{ }^{\dagger} \text { and several types of diets stated with indications for different macronutrients (section algorithm: lifestyle } \\
\text { therapy); reducing total energy (caloric) intake should be the main component of any weight-loss intervention (grade A; BEL 1); even though the } \\
\text { macronutrient composition of meals has less impact on weight loss than adherence rates in most patients. In certain patient populations, modifying } \\
\text { macronutrient compositions may be considered to optimize adherence, eating patterns, weight loss, metabolic profiles, risk factor reduction, and/or } \\
\text { clinical outcomes (grade A; BEL 1). } \\
\text { Physical exercise: recommendations }{ }^{\dagger} \text {; resistance training should be prescribed to patients with overweight or obesity undergoing weight-loss therapy } \\
\text { to promote fat loss while preserving fat-free mass; involvement of an exercise physiologist or certified fitness professional in the care plan should be } \\
\text { considered to individualize the physical activity prescription and improve outcomes (grade A; BEL 1). } \\
\text { Behavior interventions: lifestyle therapy in patients with overweight or obesity should include behavioral interventions that enhance adherence to } \\
\text { prescriptions for a reduced-calorie meal plan and increased physical activity; behavioral lifestyle intervention and support should be intensified if } \\
\text { patients do not achieve a } 2.5 \% \text { weight loss in the first month of treatment, as early weight reduction is a key predictor of long-term weight-loss success } \\
\text { (grade A; BEL 1). } \\
\text { Degrees of evidence: Origin from American Association of Clinical Endocrinologists } 2010 \text { [68]. }\end{array}$ \\
\hline & 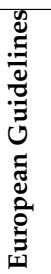 & [69] & $\begin{array}{l}\text { Dietary intervention: energy reduction }{ }^{\dagger} \text { (evidence, grade A, B); VLCDs }{ }^{\dagger} \text { are unsuitable as a sole source of nutrition for children and adolescents, } \\
\text { pregnant or lactating women and the elderly (level 2); the combination of exercise with caloric restriction helps in reducing body weight and body fat } \\
\text { and preserving FFM, as compared to diet alone (level 1; grade B) } \\
\text { Physical exercise: recommendations }{ }^{\dagger} \text { (level 2; grade B) } \\
\text { BW: A decrease of 5-15\% over a period of } 6 \text { months is realistic and of proven health benefit (evidence, level } 1)^{\dagger} \\
\text { Levels and degrees of evidence. Origin: [70]. }\end{array}$ \\
\hline \multirow[t]{2}{*}{ 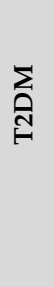 } & $\overleftrightarrow{\leftrightarrow}$ & [71] & 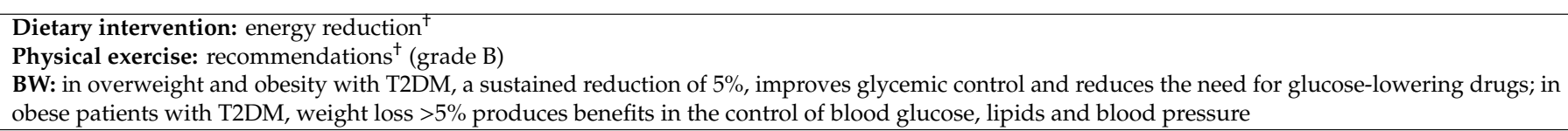 \\
\hline & 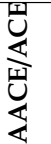 & [72] & $\begin{array}{l}\text { Lifestyle therapy: the key components are medical nutrition therapy, regular physical activity, enough hours of sleep ( } 6-9 \mathrm{~h} \text { ), behavioral support, } \\
\text { smoking cessation and avoidance of all tobacco products (this component should be left for the end of the intervention) (see Integral algorithm for the } \\
\text { management of type } 2 \text { diabetes, prediabetes and obesity). Coulston and his colleagues present another approach in the definition of "medical nutrition } \\
\text { therapy" [73]. }\end{array}$ \\
\hline \multirow[t]{2}{*}{$\stackrel{\infty}{\Sigma}$} & & [74] & $\begin{array}{l}\text { Dietary intervention and physical exercise: recommendations } \\
\text { BW: } 7-10 \% \text { reduction of body weight in a period of } 6 \text { to } 12 \text { months and achieve an ideal BMI }<25 \mathrm{~kg} \mathrm{~m}^{-2}\end{array}$ \\
\hline & 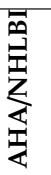 & [64] & $\begin{array}{l}\text { Dietary intervention: reduction of intake of saturated fat, trans fat, cholesterol } \\
\text { Physical exercise: recommendations }{ }^{\dagger} \\
\text { BW: } 7-10 \% \text { during the } 1 \text { year of therapy, continue with the goal of achieving a desirable weight }\left(\mathrm{BMI}<25 \mathrm{~kg} \mathrm{~m}^{-2} \text { ) }\right.\end{array}$ \\
\hline
\end{tabular}

+ Extensive information is given in Table 5. American Association of Clinical Endocrinologist (AACE); American College of Endocrinology (ACE); American Diabetes Association (ADA); American Heart Association (AHA); best evidence level (BEL); is recommended for all people with prediabetes or DM, including T1D, T2D, GDM, and other less common forms of DM. MNT must be individualized, generally via evaluation and teaching by a trained nutritionist or registered dietitian or a physician knowledgeable in nutrition (Medical Nutrition Therapy); National Heart, Lung, and Blood Institute (NHLBI); Scottish Intercollegiate Guidelines Network (SIGN); type 2 diabetes mellitus (T2DM);very low energy density diets (VLCD); kilograms $(\mathrm{Kg})$; percentage (\%); body weight (BW); minutes (min); month (mo); week (wk); pounds (lb). 
Table 5. Intervention strategies in the decrease of body composition, in overweight, obesity, type 2 diabetes mellitus and metabolic syndrome.

\begin{tabular}{|c|c|c|}
\hline \multicolumn{3}{|c|}{ Dietary Intervention } \\
\hline \multirow{3}{*}{$\begin{array}{l}\text { Energy Density Restriction/Energy } \\
\text { Density recommends }\end{array}$} & Overweight and obesity & $\pm 500-750 \mathrm{kcal} \mathrm{day}^{-1}[66] ; \pm 600 \mathrm{kcal} \mathrm{day}^{-1}[69] ; 800-1200 \mathrm{kcal} \mathrm{day}^{-1}[69]$ \\
\hline & $\mathrm{T} 2 \mathrm{DM}$ & $\begin{array}{l} \pm 500-750 \mathrm{kcal} \mathrm{day}^{-1}\left[2017 \text { [71]]/1200-1500 } \mathrm{kcal} \mathrm{day}^{-1} \text { in women and }\right. \\
1500-1800 \mathrm{kcal} \mathrm{day}^{-1} \text { in men [71] }\end{array}$ \\
\hline & MS & Reduction of 500-1000 kcal day ${ }^{-1}[74]$ \\
\hline \multirow{2}{*}{ VLCD } & Overweight and obesity & $<800 \mathrm{kcal} \mathrm{day}^{-1}[69]$ \\
\hline & T2DM and MS & ND \\
\hline \multirow[t]{2}{*}{ Macronutrients and diets } & Overweight and obesity & $\begin{array}{l}\text { Different amounts of macronutrients (hydrates, proteins and fats) and } \\
\text { giving rise to different types of diets [66]; hypocaloric balanced diets result } \\
\text { in clinically significant weight loss independent of macronutrients [69] }\end{array}$ \\
\hline & T2DM and MS & $\begin{array}{l}\text { Recommended diets may differ in advising foods high in fat or in } \\
\text { hydrates; the recommendation for consumption of recommended foods } \\
\text { does not differ: whole grains, vegetables, fruits, legumes, low-fat dairy } \\
\text { products, lean meats, nuts and seeds; it is recommended to adapt the diet } \\
\text { to the health status and the preferences of the patient }[64,71,74]\end{array}$ \\
\hline \multicolumn{3}{|c|}{ Physical Exercise } \\
\hline \multicolumn{2}{|c|}{ Overweight and obesity } & $\begin{array}{l}\text { Aerobic exercise }>150 \operatorname{min~wk}^{-1} \text { ( } 3 \text { to } 5 \text { days }^{-1} \mathrm{w}^{-1} \text { ) [67]; moderate aerobic } \\
\text { exercise: } 150 \mathrm{~min} \mathrm{wk}^{-1} \text { (energetically walking) [69] }\end{array}$ \\
\hline \multicolumn{2}{|c|}{$\mathrm{T} 2 \mathrm{DM}$} & $\begin{array}{l}\text { Greater than or equal to } 150 \mathrm{~min} \text { of physical exercise a week at moderate } \\
\text { intensity, } 3 \text { days a week at least; In the US Department of Health, does not } \\
\text { differentiate between indications for T1DM and T2DM [71]; } 150 \mathrm{~min} \mathrm{wk}^{-1} \\
\text { moderate effort; strength training and increase according to each } \\
\text { individual [72] }\end{array}$ \\
\hline \multicolumn{2}{|c|}{ MS } & $\begin{array}{l}\geq 30 \text { to } 60 \mathrm{~min} \text { of exercise (moderate intensity on most days of the week, } \\
\text { according to each individual) [74]; } \geq 30 \text { min from } 5 \text { days wk }{ }^{-1} \text { continuous } \\
\text { or intermittent (and preferably } \geq 60 \text { min and moderate intensity) [ } 64]\end{array}$ \\
\hline
\end{tabular}

Metabolic syndrome (MS); minutes (min); not stated (ND); type 1 diabetes mellitus (T1DM); type 2 diabetes mellitus (T2DM); week (wk). 
The reasoning of using the most appropriate practical guidance for obesity and T2DM in order to address the lack of knowledge in MS is: the appearance of abdominal obesity is the most frequent in this syndrome [13,75-77], and the known relationship of developing this syndrome at the same time as diabetes $[14,15,78]$. With all this in mind and as result of this work, the following advices for the design of efficient interventions in adults with MS are proposed:

To obtain the greatest changes produced in body composition, it is recommended multidisciplinary interventions (Tables 2 and 3) and Table 4 indicates 5 factors that should be intervened: medical nutrition therapy, regular physical activity, sleeping between 6 and $9 \mathrm{~h}$ sleep, limiting alcohol intake to moderate, and cessation of smoking (at the end of the procedure).

Several options have been identified in the energy constraint to be applied to dietary intake: $40 \%$ energy reduction progressively [39] or a restriction of $500 \mathrm{kcal} \mathrm{day}^{-1}$ [58] (Table 3) vs. restriction of $1000 \mathrm{kcal} \mathrm{day}^{-1}$ and energy intake (1200-1500 kcal day ${ }^{-1}$ in women and 1500-1800 kcal day ${ }^{-1}$ in men) (Table 5). Considering all the reports' approaches, we suggest a restriction between 500 and $1000 \mathrm{kcal}$ day $^{-1}$. The nutritionist should be cautious in the degree of energy restriction that is prescribed and adapt to the individualization of each subject, because there are different tolerances to fasting situations. Individuals with insulin-resistant excess weight compared with thin subjects have metabolic inflexibility (they did not register this adaptation to use fuel for the oxidation of fatty acids). This inflexibility is associated with several pathologies (metabolic syndrome, diabetes mellitus type 2 and cancer) and several factors such as: composition of the diet, frequency of ingestion, physical exercise and the use of certain pharmacological compounds $[79,80]$.

Two options are presented in the distribution of macronutrients: (i) 55-58\% carbohydrates, less than $30 \%$ fat and $15-22 \%$ proteins [39,43]; (ii) low in carbohydrates with a low glycemic index [58] (Table 3) vs. advices summarized in Table 5.

Aerobic exercise between $65-85 \%$ intensity daily (maximum heart rate) is recommended $[39,44]$ (Table 3) vs. guidelines (Table 5) which recommend $150 \mathrm{~min} \mathrm{wk}^{-1}$ (minutes per week) ( 3 to 5 days $\mathrm{wk}^{-1}$ ) and introduces strength training. The prescription for physical exercise must be structured (Table 5) [22]. The concepts of structured physical exercise were identified by the American College of Sports Medicine [81].

The reduction of $5 \%$ of body weight is advised, which will lead to improvements in metabolic alterations [30,82]. Despite the above, it would be more advisable to indicate the variation of $5 \%$ of body fat instead, in subjects with obesity and/or MS. This limit would be valid for the following cases: (i) subjects performing physical exercise, for the increase of muscle mass affecting body weight; (ii) for the reduction of subjects with low excess weight or fat (overweight and/or high fat level); (iii) and to set an accessible target for subjects with higher excess weight and fat (obesity and very high fat level) [83].

On the other hand, the following complementary advices are also established:

To follow the guidelines for the design of randomized trial reports [63] and other randomized guides [84].

The nutritionist involved should be the manager of the design of the dietetic intervention $[71,85,86]$.

To incorporate body composition measuring devices for clinical and research use on MS [86,87]. The following anthropometric parameters should be used for the calculation of metabolic risk: fat mass index (fat mass height ${ }^{-2}$ ), waist circumference, abdominal diameter and body weight [19-22]. The main predictor of adverse metabolic events is visceral adipose tissue $[75,76,88,89]$.

The tools used for evaluation of the nutritional condition at the beginning and in its follow-up must be described as follows.

a. The available procedures must be identified to recognize in what type of patients and in what moment they must be applied. The variation in the use of the procedures can be due to factors as: ethnic groups, age, gender and physical limitations (in case of the use of crutches, prothesis and/or wheelchair or even in patients without toes due to amputations because of the diabetes) $[71,86]$. 
b. The use of distance or remote health care is recommendable: (i) when the patient does not reside in the same population of the center of health and/or hospital [86]; (ii) in case of older people or specific cases in which the patients find hard to attend themselves to the center [86]; (iii) in coaching strategy [90,91].

c. Monitoring of physical exercise and/or daily physical activity is recommended (triaxial accelerometers) [92].

d. It is recommended to extrapolate the concept "obesogenic" for MS patients. This concept was firstly used in 1996 ("obese" means excess of corporal fat and "genic" refers to production or synthesis) [93]. Currently, the obesogenic environmental is defined as "the addition of the influences that have the environments, the opportunities or the living conditions to promote the obesity in individuals or populations" [94]. These influences promote the consumption of high levels or energy as well as diets based on non-healthy food (fast food, take away meals, etc.) [95].

e. Coaching is highly recommended as part of the design of further clinical trials with MS patients. In the coaching per pairs approach during interventions of overweight, obesity and diabetes patients, several instructed patients are selected to accompany and monitor to other patients suffering the same pathology. Some recent studies have revealed that thanks to the coaching per pairs approach, the levels of glycosylated hemoglobin, cholesterol, LDL, blood pressure, corporal mass index (CMI), relation waist-hip, selfcare activities in the case of diabetes, depression and other quality life factors are positively modified [90,91,96,97].

f. The nutritionist should be focused on "how" the patient or the family can apply the recommendations given by professionals. The intervention must be considered not only for the professional but also for the patient as a dynamic process, in which it will be necessary to overcome unforeseen challenges [86,98].

As a new contribution, the authors of this work extrapolate the theory of the training cycle program of an athlete to the terminology in this process of learning how to apply to lifestyle modification, improving theoretical and practical applications $[99,100]$. The learning system of the improvement of the alimentary habits must turn into a learning in the modification of the style of life (diet and exercise). In this system, three periods or blocks are differentiated (initiation, improvement and maintenance) (Table 6).

The professional should locate a subject in one period or another as follows. In the initiation period, three cases could be possible: (a) The treatment has just begun; (b) the patient is reincorporated to this period because they have increased body fat in several consecutive consultations; (c) the patient rejoins because the treatment was previously abandoned. Advancing or remaining in the improvement period occurs in two cases: a) they are in the initiation stage and body fat is decreasing regularly until there is a considerable decrease $(\geq 5 \%)$, depending on each case; $(b)$ the patient rejoins after the maintenance period because for personal reasons the treatment was paused at this stage. They will advance or remain in the maintenance period in the event that they have assimilated the recommendations to modify your lifestyle and is aware that they should not return to the previous habits in order not to relapse into the variation of body fat, having or not having reached healthy body fat level ( $\geq 10 \%$, cumulative of the initiation and improvement stage), depending on each case [86]. 
Table 6. Theory of the extrapolation of training cycle programs to the modification of lifestyle changes in metabolic syndrome with goals of body composition.

\begin{tabular}{|c|c|c|c|c|}
\hline $\begin{array}{l}\text { Lifestyle Modification } \\
\text { Program [72] }\end{array}$ & Duration & $\begin{array}{l}\text { Body Composition vs. } \\
\text { Duration }[83,98]\end{array}$ & Comments [86] & $\begin{array}{l}\text { Initial Assessment and } \\
\text { Monitoring Questionnaires }\end{array}$ \\
\hline Multiannual & Several years (2-4 years) & $\begin{array}{l}\text { Must depend on excess of weight } \\
\text { (corresponding to body fat). }\end{array}$ & $\begin{array}{l}\text { In this program, the subject may } \\
\text { evolve from one stage to another } \\
\text { (initiation, improvement } \\
\text { or maintenance). }\end{array}$ & $\begin{array}{l}\text { Activity or exercise } \\
\text { measurement: Triaxial } \\
\text { accelerometers are } \\
\text { recommended [92] } \\
\text { Food ingestion [101,102] } \\
\text { Alcohol consumption [103] }\end{array}$ \\
\hline Macrocycle & Several months & $\begin{array}{l}\text { Considerable reduction in body fat per } \\
\text { stage }(\geq 5 \%) \text {. }\end{array}$ & $\begin{array}{l}\text { Each macrocycle must be identified } \\
\text { by one stage, therefore one year may } \\
\text { have several macrocycles. }\end{array}$ & $\begin{array}{l}\text { To apply questionnaires or } \\
\text { measuring instruments as much } \\
\text { as necessary }\end{array}$ \\
\hline Mesocycle & Several weeks & $\begin{array}{l}\text { Objectives of body fat variation should } \\
\text { initially be measured by the kilos, but } \\
\text { over time, the percentage should be } \\
\text { used as the most recommended unit } \\
\text { of measure }\end{array}$ & $\begin{array}{l}\text { Weekly planning: a. variation in } \\
\text { energy intake, macronutrients; b. in } \\
\text { physical exercise (Table 5) [81]; c. } \\
\text { sleep quality; d. alcohol } \\
\text { consumption. }\end{array}$ & \\
\hline Microcycle & Several days & $\begin{array}{l}\text { It is not recommended to use body } \\
\text { composition measuring instruments. }\end{array}$ & $\begin{array}{l}\text { It is important to measure dietary } \\
\text { intake, physical exercise } \\
\text { characteristics (Table 5), sleep hours } \\
\text { and alcohol consumption (extra } \\
\text { caloric intake) }\end{array}$ & \\
\hline $\begin{array}{l}\text { Routine of one or more } \\
\text { consultation sessions }\end{array}$ & Several hours and minutes & $\begin{array}{l}\text { The evolution of body composition, } \\
\text { body fat and other anthropometric } \\
\text { parameters will be analyzed. }\end{array}$ & $\begin{array}{l}\text { A break in training lasting more than } \\
40 \text { min qualifies as two } \\
\text { separate workouts. }\end{array}$ & \\
\hline
\end{tabular}

Extrapolation [99,100]. 


\section{Conclusions}

The discrepancies found in the recommendations reported in previous works indicate that more research is required in this area of knowledge. From this systematic review, it can be concluded that the best anthropometric parameters and units of measurement to monitor the interventions are those related to dietary and physical exercise interventions. Besides, new practical guidance and advices are introduced in order to improve further clinical interventions. Thus, this systematic review will help with daily clinical practice in public health, sport sciences, nutrition and dietetics as well as endocrinology and metabolism, because the practical guidance here proposed guarantee the best results in changes of body composition, being easy to implement in daily practice in the consultation. It will therefore help the professional and patient to identify easily and quickly how the treatment should develop and what characteristics it should have. In addition, they are practical advices established based on studies in which the best results are obtained in terms of changes in body composition. The implementation of practical advices will help the public health on a large scale if the decrease of the body composition also diminishes the metabolic alterations (thus lowering the cost of healthcare).

Author Contributions: E.A.P.: collaborated in the selection of included clinical trials; elaboration of the tables and the flow diagram. M.P.G.: collaborated in the selection of included clinical trials; elaboration of the tables and the flow diagram. R.M.M.-E.: selection of the information of interest in the tables; information analysis using the PRISMA methodology; writing, translation and revision of the manuscript. M.D.M.V: selection of information of interest displayed in the tables; information analysis using the PRISMA methodology; writing, translation and revision of the manuscript. M.R.G.-G.: collaborated in the selection of the included clinical trials; elaboration and design of the tables; information analysis using the PRISMA methodology; writing of the manuscript draft.

Funding: This research has been partially funded by “Generalitat Valenciana”, Grant GV/2017/177 and VIGROB-309 (University of Alicante).

Conflicts of Interest: The authors report no conflict of interest.

\section{References}

1. Kaur, J. A Comprehensive Review on Metabolic Syndrome. Cardiol. Res. Pract. 2014, 2014, 943162. [CrossRef] [PubMed]

2. Grundy, S.M. Metabolic syndrome update. Trends Cardiovasc. Med. 2016, 26, 364-373. [CrossRef] [PubMed]

3. Duprez, D.; Toleuova, A. Prehypertension and the cardiometabolic syndrome: Pathological and clinical consequences. Expert Rev. Cardiovasc. Ther. 2013, 11, 1725-1733. [CrossRef] [PubMed]

4. American Diabetes Association. 2. Classification and Diagnosis of Diabetes. Diabetes Care 2016, 39, 13-22. [CrossRef] [PubMed]

5. Pucci, G.; Alcidi, R.; Tap, L.; Battista, F.; Mattace-Raso, F.; Schillaci, G. Sex- and gender-related prevalence, cardiovascular risk and therapeutic approach in metabolic syndrome: A review of the literature. Pharmacol. Res. 2017, 120, 34-42. [CrossRef] [PubMed]

6. Bekkouche, L.; Bouchenak, M.; Malaisse, W.; Yahia, D. The Mediterranean Diet Adoption Improves Metabolic, Oxidative, and Inflammatory Abnormalities in Algerian Metabolic Syndrome Patients. Horm. Metab. Res. 2014, 46, 274-282. [CrossRef] [PubMed]

7. Jonsdottir, S.E.; Brader, L.; Gunnarsdottir, I.; Kally Magnusdottir, O.; Schwab, U.; Kolehmainen, M.; Risérus, U.; Herzig, K.H.; Cloetens, L.; Helgegren, H.; et al. Adherence to the Nordic Nutrition Recommendations in a Nordic population with metabolic syndrome: High salt consumption and low dietary fibre intake (The SYSDIET study). Food Nutr. Res. 2013, 57. [CrossRef] [PubMed]

8. $\quad$ Alberti, K.G.; Eckel, R.H.; Grundy, S.M.; Zimmet, P.Z.; Cleeman, J.I.; Donato, K.A.; Fruchart, J.C.; James, W.P.; Loria, C.M.; Smith, S.C., Jr.; et al. Harmonizing the metabolic syndrome: A joint interim statement of the International Diabetes Federation Task Force on Epidemiology and Prevention; National Heart, Lung, and Blood Institute; American Heart Association; World Heart Federation; International Atherosclerosis Society; and International Association for the Study of Obesity. Circulation 2009, 120, 1640-1645. [CrossRef] [PubMed]

9. Martinez-Gonzalez, M.A.; Martín-Calvo, N. The major European dietary patterns and metabolic syndrome. Rev. Endocr. Metab. Disord. 2013, 14, 265-271. [CrossRef] [PubMed] 
10. Weiss, R.; Bremer, A.A.; Lustig, R.H. What is metabolic syndrome, and why are children getting it? Ann. N. Y. Acad. Sci. 2013, 1281, 123-140. [CrossRef]

11. Pérez, E.A.; Olivares, V.M.; Martínez-Espinosa, R.M.; Vila, M.D.M.; García-Galbis, M.R. New Insights about How to Make an Intervention in Children and Adolescents with Metabolic Syndrome: Diet, Exercise vs. Changes in Body Composition. A Systematic Review of RCT. Nutrients 2018, 10, 878. [CrossRef]

12. Alicka, M.; Marycz, K. The Effect of Chronic Inflammation and Oxidative and Endoplasmic Reticulum Stress in the Course of Metabolic Syndrome and Its Therapy. Stem Cells Int. 2018, 2018, 4274361. [CrossRef] [PubMed]

13. Dominguez, L.J.; Barbagallo, M. The biology of the metabolic syndrome and aging. Curr. Opin. Clin. Nutr. Metab. Care 2016, 19, 5-11. [CrossRef] [PubMed]

14. Rodríguez-Monforte, M.; Sánchez, E.; Barrio, F.; Costa, B.; Flores-Mateo, G. Metabolic syndrome and dietary patterns: A systematic review and meta-analysis of observational studies. Eur. J. Nutr. 2016, 56, 925-947. [CrossRef] [PubMed]

15. Hong, A.R.; Lim, S. Clinical characteristics of metabolic syndrome in Korea, and its comparison with other Asian countries. J. Diabetes Investig. 2015, 6, 508-515. [CrossRef] [PubMed]

16. Calton, E.K.; James, A.P.; Pannu, P.K.; Soares, M.J. Certain dietary patterns are beneficial for the metabolic syndrome: Reviewing the evidence. Nutr. Res. 2014, 34, 559-568. [CrossRef] [PubMed]

17. Yu, D.; Zhang, X.; Xiang, Y.-B.; Yang, G.; Li, H.; Gao, Y.-T.; Zheng, W.; Shu, X.-O. Adherence to dietary guidelines and mortality: A report from prospective cohort studies of 134,000 Chinese adults in urban Shanghai. Am. J. Clin. Nutr. 2014, 100, 693-700. [CrossRef]

18. Golbidi, S.; Laher, I. Exercise Induced Adipokine Changes and the Metabolic Syndrome. J. Diabetes Res. 2014, 2014, 726861. [CrossRef] [PubMed]

19. Jukarainen, S.; Holst, R.; Dalgård, C.; Piirilä, P.; Lundbom, J.; Hakkarainen, A.; Lundbom, N.; Rissanen, A.; Kaprio, J.; Kyvik, K.O; et al. Cardiorespiratory Fitness and Adiposity as Determinants of Metabolic Health-Pooled Analysis of Two Twin Cohorts. J. Clin. Endocrinol. Metab. 2017, 102, 1520-1528. [CrossRef]

20. Smith, U. Abdominal obesity: A marker of ectopic fat accumulation. J. Clin. Investig. 2015, 125, 1790-1792. [CrossRef]

21. Vasques, A.C.J.; Cassani, R.S.L.; E Forti, A.C.; Vilela, B.S.; Pareja, J.C.; Tambascia, M.A.; Geloneze, B.; BRAMS Investigators. Sagittal Abdominal Diameter as a Surrogate Marker of Insulin Resistance in an Admixtured Population-Brazilian Metabolic Syndrome Study (BRAMS). PLoS ONE 2015, 10, e0125365. [CrossRef] [PubMed]

22. Kroeger, C.M.; Hoddy, K.K.; Varady, K.A. Impact of Weight Regain on Metabolic Disease Risk: A Review of Human Trials. J. Obes. 2014, 2014, 614519. [CrossRef] [PubMed]

23. Liberati, A.; Altman, D.G.; Tetzlaff, J.; Mulrow, C.; Gøtzsche, P.C.; Ioannidis, J.P.A.; Clarke, M.; Devereaux, P.J.; Kleijnen, J.; Moher, D. The PRISMA statement for reporting systematic reviews and meta-analyses of studies that evaluate health care interventions: Explanation and elaboration. PLoS Med. 2009, 6, e1000100. [CrossRef]

24. Wiesman, F.; Hasman, A.; Herik, H.V.D. Information retrieval: An overview of system characteristics. Int. J. Med Inform. 1997, 47, 5-26. [CrossRef]

25. Salas-Salvadó, J.; Rubio, M.A.; Barbany, M.; Moreno, B. SEEDO 2007 Consensus for the evaluation of overweight and obesity and the establishment of therapeutic intervention criteria. Med. Clín. 2007, 128, 184-196.

26. Mohammadi-Sartang, M.; Bellissimo, N.; De Zepetnek, J.O.T.; Brett, N.R.; Mazloomi, S.M.; Fararouie, M.; Bedeltavana, A.; Famouri, M.; Mazloom, Z. The effect of daily fortified yogurt consumption on weight loss in adults with metabolic syndrome: A 10-week randomized controlled trial. Nutr. Metab. Cardiovasc. Dis. 2018, 28, 565-574. [CrossRef]

27. Jakubowicz, D.; Barnea, M.; Wainstein, J.; Froy, O. High Caloric intake at breakfast vs. dinner differentially influences weight loss of overweight and obese women. Obesity 2013, 21, 2504-2512. [CrossRef]

28. Casas-Agustench, P.; López-Uriarte, P.; Bulló, M.; Ros, E.; Cabré-Vila, J.; Salas-Salvadó, J. Effects of one serving of mixed nuts on serum lipids, insulin resistance and inflammatory markers in patients with the metabolic syndrome. Nutr. Metab. Cardiovasc. Dis. 2011, 21, 126-135. [CrossRef] 
29. Muzio, F.; Mondazzi, L.; Harris, W.S.; Sommariva, D.; Branchi, A. Effects of moderate variations in the macronutrient content of the diet on cardiovascular disease risk factors in obese patients with the metabolic syndrome. Am. J. Clin. Nutr. 2007, 86, 946-951. [CrossRef]

30. Mora-Rodriguez, R.; Ortega, J.F.; Morales-Palomo, F.; Ramirez-Jimenez, M. Weight loss but not gains in cardiorespiratory fitness after exercise-training predicts improved health risk factors in metabolic syndrome. Nutr. Metab. Cardiovasc. Dis. 2018, 28, 1267-1274. [CrossRef]

31. Irving, B.A.; Weltman, J.Y.; Patrie, J.T.; Davis, C.K.; Brock, D.W.; Swift, D.; Barrett, E.J.; Gaesser, G.A.; Weltman, A. Effects of Exercise Training Intensity on Nocturnal Growth Hormone Secretion in Obese Adults with the Metabolic Syndrome. J. Clin. Endocrinol. Metab. 2009, 94, 1979-1986. [CrossRef] [PubMed]

32. Irving, B.A.; Davis, C.K.; Brock, D.W.; Weltman, J.Y.; Swift, D.; Barrett, E.J.; Gaesser, G.A.; Weltman, A. Effect of exercise training intensity on abdominal visceral fat and body composition. Med. Sci. Sports Exerc. 2008, 40, 1863-1872. [CrossRef] [PubMed]

33. Wang, Q.; Chair, S.Y.; Wong, E.M.-L. The effects of a lifestyle intervention program on physical outcomes, depression, and quality of life in adults with metabolic syndrome: A randomized clinical trial. Int. J. Cardiol. 2017, 230, 461-467. [CrossRef] [PubMed]

34. Weinstock, R.S.; Trief, P.M.; Cibula, D.; Morin, P.C.; Delahanty, L.M. Weight Loss Success in Metabolic Syndrome by Telephone Interventions: Results from the SHINE Study. J. Gen. Intern. Med. 2013, 28, 1620-1628. [CrossRef] [PubMed]

35. Nanri, A.; Tomita, K.; Matsushita, Y.; Ichikawa, F.; Yamamoto, M.; Nagafuchi, Y.; Kakumoto, Y.; Mizoue, T. Effect of Six Months Lifestyle Intervention in Japanese Men with Metabolic Syndrome: Randomized Controlled Trial. J. Occup. Health 2012, 54, 215-222. [CrossRef] [PubMed]

36. Miller, M.; Sorkin, J.D.; Mastella, L.; Sutherland, A.; Rhyne, J.; Donnelly, P.; Simpson, K.; Goldberg, A.P. Poly is more effective than monounsaturated fat for dietary management in the metabolic syndrome: The muffin study. J. Clin. Lipidol. 2016, 10, 996-1003. [CrossRef] [PubMed]

37. Mora-Rodríguez, R.; Ortega, J.F.; Guio de Prada, V.; Fernández-Elías, V.E.; Hamouti, N.; Morales-Palomo, F.; Martinez-Vizcaino, V.; Nelson, R.K. Effects of Simultaneous or Sequential Weight Loss Diet and Aerobic Interval Training on Metabolic Syndrome. Int. J. Sports Med. 2016, 37, 274-281. [CrossRef] [PubMed]

38. Hill, A.M.; Jackson, K.A.H.; Roussell, M.A.; West, S.G.; Kris-Etherton, P.M. Type and amount of dietary protein in the treatment of metabolic syndrome: A randomized controlled trial. Am. J. Clin. Nutr. 2015, 102, 757-770. [CrossRef] [PubMed]

39. Bonfanti, N.; Fernández, J.M.; Gomez-Delgado, F.; Pérez-Jiménez, F. Effect of two hypocaloric diets and their combination with physical exercise on basal metabolic rate and body composition. Nutr. Hosp. 2014, 29, 635-643.

40. Neumayr, G.; Fries, D.; Mittermayer, M.; Humpeler, E.; Klingler, A.; Schobersberger, W.; Spiesberger, R.; Pokan, R.; Schmid, P.; Berent, R. Effects of hiking at moderate and low altitude on cardiovascular parameters in male patients with metabolic syndrome: Austrian Moderate Altitude Study. Wilderness Environ. Med. 2014, 25, 329-334. [CrossRef] [PubMed]

41. Dutheil, F.; Lac, G.; LeSourd, B.; Chapier, R.; Walther, G.; Vinet, A.; Sapin, V.; Verney, J.; Ouchchane, L.; Duclos, M.; et al. Different modalities of exercise to reduce visceral fat mass and cardiovascular risk in metabolic syndrome: The RESOLVE randomized trial. Int. J. Cardiol. 2013, 168, 3634-3642. [CrossRef] [PubMed]

42. Landaeta-Díaz, L.; Fernández, J.M.; Da Silva-Grigoletto, M.; Rosado-Alvarez, D.; Gómez-Garduño, A.; Gómez-Delgado, F.; López-Miranda, J.; Pérez-Jiménez, F.; Fuentes-Jiménez, F. Mediterranean diet, moderate-to-high intensity training, and health-related quality of life in adults with metabolic syndrome. Eur. J. Prev. Cardiol. 2013, 20, 555-564. [CrossRef] [PubMed]

43. Malin, S.K.; Niemi, N.; Solomon, T.P.; Haus, J.M.; Kelly, K.R.; Filion, J.; Rocco, M.; Kashyap, S.R.; Barkoukis, H.; Kirwan, J.P. Exercise training with weight loss and either a high or low glycemic diet reduces metabolic syndrome severity in older adults. Ann. Nutr. Metab. 2012, 61, 135-141. [CrossRef] [PubMed]

44. Straznicky, N.E.; Lambert, E.A.; Grima, M.T.; Eikelis, N.; Nestel, P.J.; Dawood, T.; Schlaich, M.P.; Masuo, K.; Chopra, R.; Sari, C.I.; et al. The effects of dietary weight loss with or without exercise training on liver enzymes in obese metabolic syndrome subjects. Diabetes Obes. Metab. 2012, 14, 139-148. [CrossRef] [PubMed] 
45. Wood, R.J.; Gregory, S.M.; Sawyer, J.; Milch, C.M.; Matthews, T.D.; Headley, S.A. Preservation of Fat-Free Mass after Two Distinct Weight Loss Diets with and without Progressive Resistance Exercise. Metab. Syndr. Relat. Disord. 2012, 10, 167-174. [CrossRef] [PubMed]

46. Seligman, B.G.; Polanczyk, C.A.; Santos, A.S.; Foppa, M.; Junges, M.; Bonzanini, L.; Nicolaidis, G.; Camey, S.; Lopes, A.L.; Sehl, P.; et al. Intensive practical lifestyle intervention improves endothelial function in metabolic syndrome independent of weight loss: A randomized controlled trial. Metabolism 2011, 60, 1736-1740. [CrossRef]

47. Straznicky, N.E.; Grima, M.T.; Lambert, E.A.; Eikelis, N.; Dawood, T.; Lambert, E.; Nestel, P.J.; Masuo, K.; Sari, C.I.; Chopra, R.; et al. Exercise augments weight loss induced improvement in renal function in obese metabolic syndrome individuals. J. Hypertens. 2011, 29, 553-564. [CrossRef]

48. Lerman, R.H.; Minich, D.M.; Darland, G.; Lamb, J.J.; Chang, J.-L.; Hsi, A.; Bland, J.S.; Tripp, M.L. Subjects with elevated LDL cholesterol and metabolic syndrome benefit from supplementation with soy protein, phytosterols, hops rho iso-alpha acids, and Acacia nilotica proanthocyanidins. J. Clin. Lipidol. 2010, 4, 59-68. [CrossRef]

49. Straznicky, N.E.; Lambert, E.A.; Nestel, P.J.; McGrane, M.T.; Dawood, T.; Schlaich, M.P.; Masuo, K.; Eikelis, N.; de Courten, B.; Mariani, J.A.; et al. Sympathetic neural adaptation to hypocaloric diet with or without exercise training in obese metabolic syndrome subjects. Diabetes 2010, 59, 71-79. [CrossRef]

50. Straznicky, N.E.; Lambert, G.W.; McGrane, M.T.; Masuo, K.; Dawood, T.; Nestel, P.J.; Eikelis, N.; Schlaich, M.P.; Esler, M.D.; Socratous, F.; et al. Weight Loss May Reverse Blunted Sympathetic Neural Responsiveness to Glucose Ingestion in Obese Subjects with Metabolic Syndrome. Diabetes 2009, 58, 1126-1132. [CrossRef]

51. Yassine, H.N.; Marchetti, C.M.; Krishnan, R.K.; Vrobel, T.R.; Gonzalez, F.; Kirwan, J.P. Effects of Exercise and Caloric Restriction on Insulin Resistance and Cardiometabolic Risk Factors in Older Obese Adults-A Randomized Clinical Trial. J. Gerontol. Ser. A Boil. Sci. Med. Sci. 2009, 64, 90-95. [CrossRef]

52. Katcher, H.I.; Legro, R.S.; Kunselman, A.R.; Gillies, P.J.; Demers, L.M.; Bagshaw, D.M.; Kris-Etherton, P.M. The effects of a whole grain-enriched hypocaloric diet on cardiovascular disease risk factors in men and women with metabolic syndrome. Am. J. Clin. Nutr. 2008, 87, 79-90. [CrossRef] [PubMed]

53. Chaiyasoot, K.; Sarasak, R.; Pheungruang, B.; Dawilai, S.; Pramyothin, P.; Boonyasiri, A.; Supapueng, O.; Jassil, F.C.; Yamwong, P.; Batterham, R.L. Evaluation of a 12-week lifestyle education intervention with or without partial meal replacement in Thai adults with obesity and metabolic syndrome: A randomised trial. Nutr. Diabetes 2018, 8, 23. [CrossRef] [PubMed]

54. Jahangiry, L.; Montazeri, A.; Najafi, M.; Yaseri, M.; Farhangi, M.A. An interactive web-based intervention on nutritional status, physical activity and health-related quality of life in patient with metabolic syndrome: A randomized-controlled trial (The Red Ruby Study). Nutr. Diabetes 2017, 7, e240. [CrossRef]

55. Fappa, E.; Yannakoulia, M.; Ioannidou, M.; Skoumas, Y.; Pitsavos, C.; Stefanadis, C. Telephone counselling intervention improves dietary habits and metabolic parameters of patients with the metabolic syndrome: A randomized controlled trial. Rev. Diabet. Stud. 2012, 9, 36-45. [CrossRef] [PubMed]

56. Saboya, P.P.; Bodanese, L.C.; Zimmermann, P.R.; Gustavo, A.D.; Macagnan, F.E.; Feoli, A.P.; Oliveira, M.D. Lifestyle Intervention on Metabolic Syndrome and its Impact on Quality of Life: A Randomized Controlled Trial. Arq. Bras. Cardiol. 2017, 108, 60-69. [CrossRef] [PubMed]

57. Gomez-Huelgas, R.; Jansen-Chaparro, S.; Baca-Osorio, A.; Mancera-Romero, J.; Tinahones, F.; Bernal-Lopez, M. Effects of a long-term lifestyle intervention program with Mediterranean diet and exercise for the management of patients with metabolic syndrome in a primary care setting. Eur. J. Intern. Med. 2015, 26, 317-323. [CrossRef]

58. Luley, C.; Blaik, A.; Götz, A.; Kicherer, F.; Kropf, S.; Isermann, B.; Stumm, G.; Westphal, S. Weight Loss by Telemonitoring of Nutrition and Physical Activity in Patients with Metabolic Syndrome for 1 Year. J. Am. Coll. Nutr. 2014, 33, 363-374. [CrossRef]

59. Fuller, N.R.; Pearson, S.; Lau, N.S.; Wlodarczyk, J.; Halstead, M.B.; Tee, H.-P.; Chettiar, R.; Kaffes, A.J.; Tee, H. An intragastric balloon in the treatment of obese individuals with metabolic syndrome: A randomized controlled study. Obesity 2013, 21, 1561-1570. [CrossRef]

60. Fappa, E.; Yannakoulia, M.; Skoumas, Y.; Valourdou, S.; Panagiotakos, D.B.; Pitsavos, C.; Stefanadis, C. Promoting only the consumption of healthy foods may be an alternative strategy for treating patients with the metabolic syndrome. Metabolism 2012, 61, 1361-1369. [CrossRef] 
61. Oh, E.G.; Bang, S.Y.; Hyun, S.S.; Kim, S.H.; Chu, S.H.; Jeon, J.Y.; Im, J.-A.; Lee, M.K.; Lee, J.E. Effects of a 6-month lifestyle modification intervention on the cardiometabolic risk factors and health-related qualities of life in women with metabolic syndrome. Metabolism 2010, 59, 1035-1043. [CrossRef] [PubMed]

62. Oh, E.-G.; Hyun, S.S.; Kim, S.H.; Bang, S.-Y.; Chu, S.H.; Jeon, J.Y.; Kang, M.S. A randomized controlled trial of therapeutic lifestyle modification in rural women with metabolic syndrome: A pilot study. Metabolism 2008, 57, 255-261. [CrossRef] [PubMed]

63. Moher, D.; Hopewell, S.; Schulz, K.F.; Montori, V.; Gøtzsche, P.C.; Devereaux, P.; Elbourne, D.; Egger, M.; Altman, D.G. CONSORT 2010 explanation and elaboration: Updated guidelines for reporting parallel group randomised trials. Int. J. Surg. 2012, 10, 28-55. [CrossRef] [PubMed]

64. Grundy, S.M.; Cleeman, J.I.; Daniels, S.R.; Donato, K.A.; Eckel, R.H.; Franklin, B.A.; Gordon, D.J.; Krauss, R.M.; Savage, P.J.; Smith, S.C.; et al. Diagnosis and management of the metabolic syndrome: An American Heart Association/National Heart, Lung, and Blood Institute scientific statement. Circulation 2005, 112, 2735-2752. [CrossRef] [PubMed]

65. Royall, D.; Brauer, P.; Bjorklund, L.; O’Young, O.; Tremblay, A.; Jeejeebhoy, K.; Heyland, D.; Dhaliwal, R.; Klein, D.; Mutch, D.M. Development of a Dietary Management Care Map for Metabolic Syndrome. Can. J. Diet. Pract. Res. 2014, 75, 132-139. [CrossRef] [PubMed]

66. De Lorgeril, M. Commentary on the clinical management of metabolic syndrome: Why a healthy lifestyle is important. BMC Med. 2012, 10, 139. [CrossRef] [PubMed]

67. Garvey, W.T.; Mechanick, J.I.; Brett, E.M.; Garber, A.J.; Hurley, D.L.; Jastreboff, A.M.; Nadolsky, K.; Pessah-Pollack, R.; Plodkowski, R. Reviewers of the AACE/ACE Obesity Clinical Practice Guidelines. American Association of Clinical Endocrinologist and American College of Endocrinology Comprehensive Clinical Practice Guidelines for Medical Care of Patients with obesity. Endocr. Pract. 2016, 22, 1-203. [CrossRef]

68. Mechanick, J.I.; Camacho, P.M.; Cobin, R.H.; Garber, A.J.; Garber, J.R.; Gharib, H.; Petak, S.M.; Rodbard, H.W.; Trence, D.L. American Association of Clinical Endocrinologists Protocol for Standardized Production of Clinical Practice Guidelines-2010 Update. Endocr. Pract. 2010, 16, 270-283. [CrossRef]

69. Yumuk, V.; Tsigos, C.; Fried, M.; Schindler, K.; Busetto, L.; Micic, D.; Toplak, H. European Guidelines for Obesity Management in Adults. Obes. Facts 2015, 8, 402-424. [CrossRef]

70. Scottish Intercollegiate Guidelines Network. Obesity in Scotland. Integrating Prevention with Weight Management. A National Clinical Guideline Recommended for Use in Scotland; Scottish Intercollegiate Guidelines Network: Edinburgh, Scotland, 1996.

71. American Diabetes Association. 4. Lifestyle Management. Diabetes Care 2017, 40, 33-43. [CrossRef]

72. Garber, A.J.; Abrahamson, M.J.; Barzilay, J.I.; Blonde, L.; Bloomgarden, Z.T.; Bush, M.A.; Dagogo-Jack, S.; DeFronzo, R.A.; Einhorn, D.; Fonseca, V.A.; et al. Consensus Statement by the American Association of Clinical Endocrinologists and American College of Endocrinology on the Comprehensive Type 2 Diabetes Management Algorithm-2018 Executive Summary. Endocr. Pract. 2018, 24, 91-120. [CrossRef]

73. Coulston, A.M.; Rosen, C. American health care reform: Implications for medical nutrition therapy. Am. J. Clin. Nutr. 1994, 59, 1275-1276. [CrossRef] [PubMed]

74. Champagne, C.M.; Bray, G.A. Dietary management of the metabolic syndrome-One size fits all? Proc. Nutr. Soc. 2013, 72, 310-316. [CrossRef] [PubMed]

75. Misra, A.; Soares, M.J.; Mohan, V.; Anoop, S.; Abhishek, V.; Vaidya, R.; Pradeepa, R. Body fat, metabolic syndrome and hyperglycemia in South Asians. J. Diabetes Complicat. 2018, 32, 1068-1075. [CrossRef] [PubMed]

76. Armani, A.; Berry, A.; Cirulli, F.; Caprio, M. Molecular mechanisms underlying metabolic syndrome: The expanding role of the adipocyte. FASEB J. 2017, 31, 4240-4255. [CrossRef] [PubMed]

77. Engin, A. The Definition and Prevalence of Obesity and Metabolic Syndrome. Adv. Exp. Med. Biol. 2017, 960, 1-17. [PubMed]

78. Grundy, S.M. Adipose tissue and metabolic syndrome: Too much, too little or neither. Eur. J. Clin. Investig. 2015, 45, 1209-1217. [CrossRef] [PubMed]

79. Smith, R.L.; Soeters, M.R.; Wüst, R.C.I.; Houtkooper, R.H. Metabolic Flexibility as an Adaptation to Energy Resources and Requirements in Health and Disease. Endocr. Rev. 2018, 39, 489-517. [CrossRef] 
80. Kahlhöfer, J.; Lagerpusch, M.; Skurk, T.; Müller, M.J.; Bosy-Westphal, A.; Bosy-Westphal, A. Deep body composition phenotyping during weight cycling: Relevance to metabolic efficiency and metabolic risk. Obes. Rev. 2015, 16, 36-44.

81. Garber, C.E.; Blissmer, B.; Deschenes, M.R.; Franklin, B.A.; Lamonte, M.J.; Lee, I.M.; Nieman, D.C.; Swain, D.P.; American College of Sports Medicine. American College of Sports Medicine position stand. Quantity and quality of exercise for developing and maintaining cardiorespiratory, musculoskeletal, and neuromotor fitness in apparently healthy adults: Guidance for prescribing exercise. Med. Sci. Sports Exerc. 2011, 43, 1334-1359. [CrossRef]

82. Franz, M.J.; Boucher, J.L.; Rutten-Ramos, S.; VanWormer, J.J. Lifestyle Weight-Loss Intervention Outcomes in Overweight and Obese Adults with Type 2 Diabetes: A Systematic Review and Meta-Analysis of Randomized Clinical Trials. J. Acad. Nutr. Diet. 2015, 115, 1447-1463. [CrossRef] [PubMed]

83. García-Galbis, M.R.; Baeza, M.R.; Castell, E.C. Indicators of success in the dietary management of overweight and obesity: Weight, body fat loss and quality. Nutr. Hosp. 2015, 32, 1009-1016.

84. Argimon, J.M.; Jimenéz, J. Métodos de Investigación Clínica y Epidemiológica, 4th ed.; Elsevier: Barcelona, Spain, 2013.

85. Raynor, H.A.; Davidson, P.G.; Burns, H.; Nadelson, M.D.H.; Mesznik, S.; Uhley, V.; Moloney, L. Medical Nutrition Therapy and Weight Loss Questions for the Evidence Analysis Library Prevention of Type 2 Diabetes Project: Systematic Reviews. J. Acad. Nutr. Diet. 2017, 117, 1578-1611. [CrossRef] [PubMed]

86. García-Galbis, M.R. The management of qualitative and quantitative dietary treatment for overweight and obesity: Methodology and a new perspective on individualised assessment. Nutr. Hosp. 2015, 32, 2019-2029.

87. Müller, M.J.; Braun, W.; Pourhassan, M.; Geisler, C.; Bosy-Westphal, A. Application of standards and models in body composition analysis. Proc. Nutr. Soc. 2016, 75, 181-187. [CrossRef] [PubMed]

88. Pi-Sunyer, X. Changes in body composition and metabolic disease risk. Eur. J. Clin. Nutr. 2019, 73, $231-235$. [CrossRef] [PubMed]

89. Shah, R.V.; Murthy, V.L.; Abbasi, S.A.; Blankstein, R.; Kwong, R.Y.; Goldfine, A.B.; Jerosch-Herold, M.; Lima, J.A.; Ding, J.; Allison, M.A. Visceral Adiposity and the Risk of Metabolic Syndrome Across Body Mass Index. JACC Cardiovasc. Imaging 2014, 7, 1221-1235. [CrossRef] [PubMed]

90. Aalaa, M.; Sanjari, M.; Aghaei Meybodi, H.R.; Amini, M.R.; Qorbani, M.; Adibi, H.; Mehrdad, N. The Effectiveness of a Peer Coaching Education on Control and Management of Type 2 Diabetes in Women: A Protocol for a Randomized Controlled Trial. Int. J. Community Based Nurs. Midwifery 2017, 5, 153-164.

91. Rogers, E.A.; Hessler, D.M.; Bodenheimer, T.S.; Ghorob, A.; Vittinghoff, E.; Thom, D.H. Diabetes peer coaching: Do "better patients" make better coaches? Diabetes Educ. 2014, 40, 107-115. [CrossRef] [PubMed]

92. De Toro-Martín, J.; Arsenault, B.J.; Després, J.-P.; Vohl, M.-C. Precision Nutrition: A Review of Personalized Nutritional Approaches for the Prevention and Management of Metabolic Syndrome. Nutrients 2017, 9, 913. [CrossRef]

93. Gauthier, K.I.; Krajicek, M.J. Obesogenic environment: A concept analysis and pediatric perspective. J. Spéc. Pediatr. Nurs. 2013, 18, 202-210. [CrossRef] [PubMed]

94. Cauchi, D.; Rutter, H.; Knai, C. An obesogenic island in the Mediterranean: Mapping potential drivers of obesity in Malta. Public Health Nutr. 2015, 18, 3211-3223. [CrossRef] [PubMed]

95. Giskes, K.; van Lenthe, F.; Avendano-Pabon, M.; Brug, J. A systematic review of environmental factors and obesogenic dietary intakes among adults: Are we getting closer to understanding obesogenic environments? Obes. Rev. 2011, 12, e95-e106. [CrossRef] [PubMed]

96. Pirbaglou, M.; Katz, J.; Motamed, M.; Pludwinski, S.; Walker, K.; Ritvo, P. Personal Health Coaching as a Type 2 Diabetes Mellitus Self-Management Strategy: A Systematic Review and Meta-Analysis of Randomized Controlled Trials. Am. J. Health Promot. 2018, 32, 1613-1626. [CrossRef] [PubMed]

97. Van Rinsum, C.; Gerards, S.; Rutten, G.; Philippens, N.; Janssen, E.; Winkens, B.; Van De Goor, I.; Kremers, S. The Coaching on Lifestyle (CooL) Intervention for Overweight and Obesity: A Longitudinal Study into Participants' Lifestyle Changes. Int. J. Environ. Res. Public Health 2018, 15, 680. [CrossRef] [PubMed]

98. Reig García-Galbis, M.; Rizo Baeza, M.M.; Cortés Castell, E. Predictors of weight loss and fat in the dietary management: Sex, age, BMI and consulting assistance. Nutr. Hosp. 2015, 32, 1028-1035. [CrossRef] 
99. Issurin, V.B. Benefits and Limitations of Block Periodized Training Approaches to Athletes' Preparation: A Review. Sports Med. 2016, 46, 329-338. [CrossRef]

100. Issurin, V.B. New Horizons for the Methodology and Physiology of Training Periodization. Sports Med. 2010, 40, 189-206. [CrossRef]

101. Kirkpatrick, S.I.; Vanderlee, L.; Raffoul, A.; Stapleton, J.; Csizmadi, I.; Boucher, B.A.; Massarelli, I.; Rondeau, I.; Robson, P.J. Self-Report Dietary Assessment Tools Used in Canadian Research: A Scoping Review. Adv. Nutr. 2017, 8, 276-289. [CrossRef]

102. Ortega, R.; Requejo, A.M. Nutriguía. Manuel de Nutrición Clínica, 2nd ed.; Editorial Panamericana: Madrid, España, 2015; pp. 137-152.

103. Pan American Health Organization. International Guide to Monitor Alcohol Consumption and Its Health Consequences; World Health Organization: Geneva, Switzerland, 2000; pp. 159-167. Available online: https://apps.who.int/iris/bitstream/handle/10665/66529/WHO_MSD_MSB_00.4.pdf; jsessionid=0F2662BB866105AC29BBB1B42A990576? sequence=1 (accessed on 4 September 2019).

(C) 2019 by the authors. Licensee MDPI, Basel, Switzerland. This article is an open access article distributed under the terms and conditions of the Creative Commons Attribution (CC BY) license (http://creativecommons.org/licenses/by/4.0/). 